\title{
Multiplicidade de soluções positivas para uma equação de Schrödinger não linear
}

\author{
Moreno Pereira Bonutti
}

Orientador: Sérgio Henrique Monari Soares

Dissertação apresentada ao Instituto de Ciências Matemáticas e de Computação da Universidade de São Paulo, como parte dos requisitos para obtenção do título de Mestre em Matemática.

USP - São Carlos

Janeiro/2010

\footnotetext{
${ }^{1}$ Este trabalho teve suporte financeiro da CAPES
} 

"Um raciocínio lógico leva você de $A$ a B. Imaginação leva você a qualquer lugar que você quiser."

Albert Einstein 


\section{Agradecimentos}

Agradeço primeiramente a Deus por ter me dado força para enfrentar essa jornada. Agradeço ao meu orientador Sérgio Henrique Monari Soares, um professor muito sábio e inteligente, pelos ótimos conselhos e pela imensa paciência. Sem a ajuda do professor não teria conseguido vencer esse desafio.

Obrigado a CAPES pelo financiamento dos meus estudos.

Agradeço também aos meu amigos de mestrado que me ajudaram muito durante todo o desenvolvimento deste trabalho, agradeço muito ao Matheus C. Bortolan e a Juliana T. de Lima por serem meus amigos de todas as horas e meus professores, nas intermináveis horas de estudo. Agradeço também ao meu amigo Rafael Rossato, que sempre arrumava um tempo e "matava" horas de estudos para conversar sobre qualquer assunto que não fosse a matemática.

Não posso esquecer dos meus grandes amigos da República Infiltrados, Mosquito, Nando, Farofa, Caxa e Léo que me hospedaram e me ajudaram a descontrair com muitos churrascos e sambas.

Agradeço a Nathalia Rossetti, minha grande companheira e meu grande amor, por ter me ajudado em todas as horas difíceis sem ela eu não conseguiria realizar este trabalho. Obrigado por me fazer feliz.

Por fim sou grato a minha família por ter me dado uma ótima educação e ter me ajudado a manter os meus estudos. Vocês são muito importantes na minha vida.

A todos vocês, muitíssimo obrigado!!! 


\section{Resumo}

Este trabalho é dedicado ao estudo da existência de soluções da equação de Schrödinger

$$
-\Delta u+(\lambda a(x)+1) u=u^{p}, \quad u>0 \quad \text { em } \mathbb{R}^{N},
$$

onde $a \geq 0$ é uma função contínua e $p>1$ é um expoente subcrítico. Métodos Variacionais são empregados para mostrar a existência de uma sequência $\lambda_{n} \rightarrow+\infty$ e da respectiva sequência de soluções $u_{\lambda_{n}}$ convergindo para uma solução de energia mínima do problema de Dirichlet

$$
-\Delta u+u=u^{p}, \quad u>0 \mathrm{em} \Omega, \quad u=0 \text { sobre } \partial \Omega,
$$

sendo $\Omega:=\operatorname{int} a^{-1}(0)$. Além disso, estuda-se o efeito da topologia do conjunto $\Omega$ sobre o número de soluções da equação $(*)$ por meio da categoria de Lusternik e Schnirelman. 


\section{Abstract}

This work is devoted to study the existence of positive solutions of the Schrödinger equation

$$
-\Delta u+(\lambda a(x)+1) u=u^{p}, \quad u>0 \text { in } \mathbb{R}^{N},
$$

where $a$ is a nonnegative and continuous function and $p>1$ is a subcritical exponent. Variational methods are employed in order to show the existence of a sequence $\lambda_{n} \rightarrow+\infty$ and the respective sequence of solutions converging in $H^{1}\left(\mathbb{R}^{N}\right)$ to a least energy solution of the Dirichlet problem

$$
-\Delta u+u=u^{p}, \quad u>0 \text { in } \Omega, \quad u=0 \text { on } \partial \Omega,
$$

where $\Omega:=\operatorname{int} a^{-1}(0)$. Furthermore, it is studied the effect of the topology of the set $\Omega$ on the number of positive solutions of the equation $(*)$ by using the Lusternik and Schnirelman category. 


\section{Índice}

\begin{tabular}{lll}
\hline 1 & Introdução & 1
\end{tabular}

2 Múltiplas soluções positivas

2.1 Condição de compacidade do funcional . . . . . . . . . . . . . . . 5

2.2 Prova dos Resultados Principais . . . . . . . . . . . . . . . . . 13

\begin{tabular}{|ll}
\hline A Apêndice A & 29
\end{tabular}



\begin{tabular}{lll}
\hline C Apêndice C & 41 \\
\hline
\end{tabular}

\begin{tabular}{ll}
\hline Referências bibliográficas & 44
\end{tabular} 




Neste trabalho temos como objetivo estudar a existência e multiplicidade de soluções positivas para a seguinte equação de Schöndinger não linear:

$$
-\Delta u+(\lambda a(x)+1) u=u^{p}, u>0 \mathrm{em} \mathbb{R}^{N} .
$$

As soluções positivas que encontraremos dependerão de $\lambda, p$ e do potencial $b_{\lambda}(x)=\lambda a(x)+1$. Iremos trabalhar com $N \geqslant 3$ e com o problema subcrítico, isto é, $1<p<2^{*}-1$, sendo $2^{*}=2 N /(N-2), N \geq 3$. O potencial $b_{\lambda}$ satisfaz as seguintes hipóteses:

(A1) $a \in C\left(\mathbb{R}^{N}, \mathbb{R}\right)$, satisfaz $a \geqslant 0$ e $\Omega:=\operatorname{int}\left\{a^{-1}(0)\right\}$ é não vazio com fronteira suave, $\operatorname{com} \bar{\Omega}=a^{-1}(0)$.

(A2) Existe $M_{0}>0$ tal que

$$
\mu\left(\left\{x \in \mathbb{R}^{N}: a(x) \leqslant M_{0}\right\}\right)<\infty,
$$

onde $\mu$ denota a medida de Lebesgue em $\mathbb{R}^{N}$

Para descrever os resultados principais que serão aqui abordados, considere o funcional

$$
\Phi_{\lambda . p}(u)=\frac{1}{2} \int_{\mathbb{R}^{N}}\left[|\nabla u|^{2}+(\lambda a(x)+1) u^{2}\right] d x-\frac{1}{p+1} \int_{\mathbb{R}^{N}}|u|^{p+1} d x
$$

Associado à equação $\left(\mathrm{S}_{\lambda, \mathrm{p}}\right)$, o qual está definido em

$$
E=\left\{u \in H^{1}\left(\mathbb{R}^{N}\right): \int_{\mathbb{R}^{N}} a(x) u^{2} d x<+\infty\right\} .
$$


Defina a variedade de Nehari

$$
M_{\lambda, p}=\left\{u \in E \backslash\{0\}: \int_{\mathbb{R}^{N}}\left(|\nabla u|^{2}+(\lambda a(x)+1) u^{2}\right) d x=\int_{\mathbb{R}^{N}}|u|^{p+1} d x\right\}
$$

e seja

$$
c_{\lambda, p}=\inf _{u \in M_{\lambda, p}} \Phi_{\lambda, p}(u)
$$

Em [BW00], Bartsch e Wang provaram os seguintes teoremas:

Teorema 1.0.1. Suponha que as hipóteses (A1) e (A2) estão satisfeitas. Então para $\lambda$ suficientemente grande, o infimo é atingido, e assim $\left(\mathrm{S}_{\lambda, \mathrm{p}}\right)$ possui uma solução de menor energia $u_{\lambda, p}$ correspondente ao nível $c_{\lambda, p}$. Além disso, qualquer sequência $\lambda_{n} \rightarrow+\infty$ possui uma subsequência tal que $u_{\lambda_{n}}$ converge em $H^{1}\left(\mathbb{R}^{N}\right)$, a menos de uma subsequência, para uma solução de menor energia do problema de Dirichlet,

$$
-\Delta u+u=u^{p}, u>0 \text { em } \Omega
$$

O próximo resultado mostra o efeito da topologia de $\Omega$ sobre o número de soluções positivas de $\left(\mathrm{S}_{\lambda, \mathrm{p}} \mathrm{p}\right.$.

Teorema 1.0.2. Suponha que (A1) e (A2) estão satisfeitas e $\Omega$ é limitado. Então existem $p_{0}<2^{*}-1$ e uma função $\Lambda:\left(p_{0}, 2^{*}-1\right) \rightarrow \mathbb{R}$ tal que $\left(\mathrm{S}_{\lambda, \mathrm{p}}\right)$ possui pelo menos $\operatorname{cat}(\Omega)^{1}$ soluções distintas para todo $\lambda \geqslant \Lambda(p)$.

Como no caso de soluções de energia mínima, as soluções obtidas pelo teorema anterior convergem para uma solução de energia mínima de $(\mathrm{Dp})$.

Teorema 1.0.3. Suponha que (A1) e (A2) estão satisfeitas. Seja $u_{n}$ uma sequência de soluções de $\left(\mathrm{S}_{\lambda, \mathrm{p}}\right)$, com $\lambda_{n} \rightarrow+\infty$ e $\lim \sup _{n \rightarrow+\infty} \Phi_{\lambda_{n}, p}\left(u_{n}\right)<\infty$. Entãa, a menos de uma subsequência, $u_{n}$ converge fortemente, em $H^{1}\left(\mathbb{R}^{N}\right)$ para uma solução de (Dp).

Há vários trabalhos que estudam a existência de soluções positivas para problemas relacionados à equação $\left(\mathrm{S}_{\lambda, \mathrm{p}}\right)$. Por exemplo, em [AdMFS09, Alves et all estudaram o caso com crescimento crítico para o seguinte problema

$$
-\Delta u+(\lambda V(x)+Z(x)) u=\beta u^{q}+u^{2^{*}-1}, \text { para } u>0 \text { em } \mathbb{R}^{N},
$$

onde $\lambda, \beta \in(0,+\infty), q \in\left(1,2^{*}-1\right), N \geqslant 3$ e $V, Z: \mathbb{R}^{N} \rightarrow \mathbb{R}$ são funções contínuas satisfazendo algumas hipóteses.

\footnotetext{
${ }^{1}$ Aqui $\operatorname{cat}(\Omega)$ denota a categoria de Lusternik-Schnirelmann do conjunto $\Omega$. Veja Apêndice C.
} 
Em AS08] Alves e Souto também realizaram o estudo das soluções positivas com crescimento crítico exponencial no caso $N=\mathbb{R}^{2}$ para a classe de problemas

$$
\begin{cases}-\Delta u+(\lambda V(x)+Z(x)) u=f(u), & \text { em } \mathbb{R}^{2}, \\ u>0, & \text { em } \mathbb{R}^{2}, \\ u \in H^{1}\left(\mathbb{R}^{2}\right), & \end{cases}
$$

onde $f$ é de classe $C^{1}$ com crescimento exponencial crítico, $\lambda \in(1,+\infty), V, Z: \mathbb{R}^{2} \rightarrow \mathbb{R}$ são funções contínuas com $V(x)>0$ para todo $x \in \mathbb{R}^{2} \mathrm{e} \Omega=i n t V^{-1}(0)$ possui $k$ componentes conexas denotadas por $\Omega_{j}, j \in\{1,2, \ldots k\}, V^{-1}(\{0\}=\bar{\Omega}$ e $\partial \Omega$ é suave.

Outro trabalho interessante é dD02, no qual De Figueiredo e Ding estudaram a existência e a multiplicidade das soluções do problema

$$
\left\{\begin{array}{l}
-\Delta u+\left(\lambda a(x)+a_{0}(x)\right) u=g(x, u) \\
u \in H^{1}\left(\mathbb{R}^{N}\right)
\end{array}\right.
$$

para o caso subcrítico e estudaram a existência e a multiplicidade de soluções do problema com crescimento crítico

$$
\left\{\begin{array}{l}
-\Delta u+\left(\lambda a(x)+a_{0}(x)\right) u=K(x)|u|^{2^{*}-2} u+g(x, u) \\
u \in H^{1}\left(\mathbb{R}^{N}\right) .
\end{array}\right.
$$

Em [DS07], Ding e Szulkin estudaram a existência de soluções para a equação de Schrödinger semilinear

$$
-\epsilon^{2} \Delta u+V(x) u=g(x, u)
$$

$\operatorname{com} \epsilon>o$ pequeno e

$$
-\Delta u+\lambda V(x) u=g(x, u)
$$

com $\lambda>0$ grande, onde o potencial $V$ pode mudar de sinal.

Em [CW07], Chabrowski e Wang estudaram a existência de soluções do seguinte problema de Neumann

$$
\left\{\begin{array}{lr}
-\Delta u+b(x) u=Q(x)|u|^{p-2} u & \text { em } \Omega^{c} \\
\frac{\partial}{\partial \nu} u(x)=0 & \text { em } \partial \Omega, u>0 \text { em } \Omega^{c} .
\end{array}\right.
$$

Neste trabalho, motivados por [BW00], apresentaremos as demonstrações dos teoremas 1.0.1 - 1.0.3. Para tanto, dividimos este trabalho em um capítulo e três apêndices. O Capítulo 2 é reservado para estudar a condição de compacidade do funcional associado ao problema $\left(S_{\lambda, p}\right)$ e provar os resultados principais deste trabalho seguindo os argumentos de Bartsch e Wang [BW00]. 


\section{Capítulo}

2

\section{Múltiplas soluções positivas para a equação de Schrödinger não linear}

\subsection{Condição de compacidade do funcional}

Nesta seção, consideraremos uma condição de compacidade para o funcional associado à equação

$$
\Delta u+(\lambda a(x)+1) u=f(x, u) \text { em } \mathbb{R}^{N} .
$$

No que segue, admitiremos que a função $f: \mathbb{R}^{N} \times \mathbb{R} \rightarrow \mathbb{R}$ satisfaça as seguintes hipóteses:

(A3) $f \in \mathcal{C}^{1}\left(\mathbb{R}^{N} \times \mathbb{R}, \mathbb{R}\right)$ satisfaz $f(x, u)=o(|u|)$ quando $u \rightarrow 0$ uniformemente em $x$;

(A4) Existem constantes $a_{1}, a_{2}>0$ e $1<s<2^{*}-1$ tais que

$$
|f(x, u)| \leqslant a_{1}\left(1+|u|^{s}\right) \text { e }\left|f^{\prime}(x, u)\right| \leqslant a_{2}\left(1+|u|^{s-1}\right) \text { para todo } x \in \mathbb{R}^{N}, u \in \mathbb{R}
$$

(A5) Existe $q>2$ tal que, para todo $x \in \mathbb{R}^{N}, u \in \mathbb{R} \backslash\{0\}$, temos

$$
0<q F(x, u) \equiv q \int_{0}^{u} f(x, v) d v \leqslant u f(x, u)
$$

Consideremos o conjunto $E=\left\{u \in H^{1}\left(\mathbb{R}^{N}\right): \int_{\mathbb{R}^{N}} a(x) u^{2} d x<\infty\right\}$ munido da norma 


$$
\|u\|_{\lambda}^{2}=\int_{\mathbb{R}^{N}}\left(|\nabla u|^{2}+(\lambda a(x)+1) u^{2}\right) d x \text { para } \lambda \geqslant 0 .
$$

Observemos que as normas $\|\cdot\|_{\lambda}$ para $\lambda \geqslant 0$ são equivalentes em $E$ e sabemos que as soluções de $\left(\mathrm{S}_{\lambda}\right)$ são pontos críticos do funcional $\Phi_{\lambda}: E \rightarrow \mathbb{R}$, onde

$$
\Phi_{\lambda}(u)=\frac{1}{2}\|u\|_{\lambda}^{2}-\int_{\mathbb{R}^{N}} F(x, u) d x .
$$

Observe também que por (A3) e (A4) o funcional $\Phi_{\lambda}$ está bem definido e $\Phi_{\lambda} \in C^{1}(E, \mathbb{R})$ para todo $\lambda \geqslant 0$ (ver A.0.15, A.0.18 e A.0.19 no Apêndice A).

A seguir enunciaremos o principal resultado desta seção.

Proposição 2.1.1. Suponha que as hipóteses (A1)-(A5) estejam satisfeitas. Então para todo $C_{0}>0$ existe $\Lambda_{0}>0$ tal que $\Phi_{\lambda}$ satisfaz a condição $(P S)_{c}$ para todo $\lambda \geqslant \Lambda_{0}$ e para todo $c \leqslant C_{0}$.

A fim de provarmos a Proposição 2.1.1, demonstraremos alguns lemas.

Lema 2.1.2. Seja $K_{\lambda}$ o conjunto formado pelos pontos críticos de $\Phi_{\lambda}$. Então existe $\sigma>0$ (independente de $\lambda$ ) tal que $\|u\|_{\lambda} \geqslant\|u\|_{H^{1}} \geqslant \sigma$ para todo $u \in K_{\lambda} \backslash\{0\}$.

Demonstração: da hipótese (A3), dado $\epsilon>0$, existe $u_{0}=u_{0}(\epsilon)$ tal que $|u|<u_{0}$. Então

$$
|f(x, u)| \leqslant \epsilon|u|
$$

e portanto

$$
|f(x, u) u| \leqslant \epsilon|u|^{2} .
$$

Por (A4), para todo $|u| \geqslant u_{0}$,

$$
f(x, u) u \leqslant a_{1}\left(\frac{1}{|u|^{s}}+1\right)|u|^{s+1} \leqslant a_{1}\left(\frac{1}{u_{0}^{s}}+1\right)|u|^{s+1} .
$$

Denotando $A(\epsilon)=a_{1}\left(\frac{1}{u_{0}^{s}}+1\right)$, segue que

$$
f(x, u) \leqslant \epsilon u^{2}+A(\epsilon)|u|^{s+1} \text {, para todo } x \in \mathbb{R}^{N}, u \in \mathbb{R} \text {. }
$$

Por fim, para $u \in K_{\lambda} \backslash\{0\}$ temos $\Phi_{\lambda}^{\prime}(u)(u)=0$, ou seja,

$$
0=\Phi_{\lambda}^{\prime}(u)(u)=\|u\|_{\lambda}^{2}-\int_{\mathbb{R}^{N}} f(x, u) u d x \geqslant\|u\|_{\lambda}^{2}-\int_{\mathbb{R}^{N}}\left(\epsilon|u|^{2}+A(\epsilon)|u|^{s+1}\right) d x .
$$


Observe que

$$
\int_{\mathbb{R}^{N}}\left(\epsilon|u|^{2}+A(\epsilon)|u|^{s+1}\right) d x=\epsilon\|u\|_{L^{2}\left(\mathbb{R}^{N}\right)}^{2}+A(\epsilon)\|u\|_{L^{s+1}\left(\mathbb{R}^{N}\right)}^{s+1} .
$$

Usando as imersões de Sobolev

$$
\begin{aligned}
0 \geqslant\|u\|_{\lambda}^{2}-\epsilon\|u\|_{L^{2}\left(\mathbb{R}^{N}\right)}^{2} & -A(\epsilon)\|u\|_{L^{s+1}\left(\mathbb{R}^{N}\right)}^{s+1} \\
& \geqslant\|u\|_{\lambda}^{2}-C\left[\epsilon\|u\|_{H^{1}\left(\mathbb{R}^{N}\right)}^{2}+A(\epsilon)\|u\|_{H^{1}\left(\mathbb{R}^{N}\right)}^{s+1}\right] \\
& \geqslant\|u\|_{H^{1}\left(\mathbb{R}^{N}\right)}^{2}-C \epsilon\|u\|_{H^{1}\left(\mathbb{R}^{N}\right)}^{2}-C A(\epsilon)\|u\|_{H^{1}\left(\mathbb{R}^{N}\right)}^{s+1},
\end{aligned}
$$

uma vez que $\|u\|_{\lambda}^{2} \geqslant\|u\|_{H^{1}\left(\mathbb{R}^{N}\right)}^{2}$. Assim

$$
\|u\|_{H^{1}\left(\mathbb{R}^{N}\right)}^{2}\left(1-C \epsilon-K\|u\|_{H^{1}\left(\mathbb{R}^{N}\right)}^{s-1}\right) \leqslant 0 \text {, onde } K=C A(\epsilon) .
$$

Ainda, como $\|u\|_{H^{1}\left(\mathbb{R}^{N}\right)}^{2} \geqslant 0$,

$$
\|u\|_{H^{1}\left(\mathbb{R}^{N}\right)} \geqslant\left(\frac{1-C \epsilon}{K}\right)^{\frac{1}{s-1}} \doteq \sigma .
$$

Lema 2.1.3. Existe uma constante $c_{0}>0$, independente de $\lambda$, tal que se $\left(u_{n}\right)$ é uma sequência $(P S)_{c}$ de $\Phi_{\lambda}$, então

(i) $\limsup _{n \rightarrow+\infty}\left\|u_{n}\right\|_{\lambda}^{2} \leqslant \frac{2 q c}{q-2}$;

(ii) $c \geqslant c_{0}$ ou $c=0$.

Demonstração: de $\Phi_{\lambda}^{\prime}\left(u_{n}\right) \rightarrow 0$ e $\Phi_{\lambda}\left(u_{n}\right) \rightarrow c$ segue que para $\epsilon>0$ dado, existem $n_{0} \in \mathbb{N}$ e constante $C>0$ tal que

$$
\Phi_{\lambda}\left(u_{n}\right)-\frac{1}{q} \Phi_{\lambda}^{\prime}\left(u_{n}\right)\left(u_{n}\right) \leqslant c+\epsilon+C\left\|u_{n}\right\|_{\lambda}
$$

Por outro lado, por (A5),

$$
\begin{aligned}
\Phi_{\lambda}\left(u_{n}\right)-\frac{1}{q} \Phi_{\lambda}^{\prime}\left(u_{n}\right)\left(u_{n}\right) & =\frac{q-2}{2 q}\left\|u_{n}\right\|_{\lambda}^{2}+\int_{\mathbb{R}^{N}}\left(\frac{1}{q} f\left(x, u_{n}\right) u_{n}-F\left(x, u_{n}\right)\right) d x \\
& \geqslant \frac{q-2}{2 q}\left\|u_{n}\right\|_{\lambda}^{2} .
\end{aligned}
$$

Portanto,

$$
\frac{q-2}{2 q}\left\|u_{n}\right\|_{\lambda}^{2} \leqslant c+\epsilon+C\left\|u_{n}\right\|_{\lambda}, \text { para todo } n \geqslant n_{0}
$$


logo, $\left(u_{n}\right)$ é limitado em $E$. Mais ainda, por 2.2 encontramos

$$
\frac{q-2}{2 q}\left\|u_{n}\right\|_{\lambda}^{2} \leqslant \Phi_{\lambda}\left(u_{n}\right)-\frac{1}{q} \Phi_{\lambda}^{\prime}\left(u_{n}\right)\left(u_{n}\right) \leqslant \Phi_{\lambda}\left(u_{n}\right)+\frac{1}{q}\left\|\Phi_{\lambda}^{\prime}\left(u_{n}\right)\right\|\left\|u_{n}\right\|_{\lambda} .
$$

Portanto da limitação de $\left(u_{n}\right)$ em $E$ e de $\Phi_{\lambda}\left(u_{n}\right) \rightarrow c$ segue que

$$
\limsup _{n \rightarrow+\infty}\left\|u_{n}\right\|_{\lambda}^{2} \leqslant \frac{2 q c}{q-2}
$$

Agora, por (A3)-(A5) existe $C>0$ independente de $\lambda \geqslant 0$ tal que

$$
\Phi_{\lambda}^{\prime}(u)(u)=\|u\|_{\lambda}^{2}-\int_{\mathbb{R}^{N}} f(x, u) u d x \geqslant \frac{1}{2}\|u\|_{\lambda}^{2}-C\|u\|_{\lambda}^{s+1}=\left(\frac{1}{2}-C\|u\|_{\lambda}^{s-1}\right)\|u\|_{\lambda}^{2} .
$$

Como $1<s<2^{*}-1$, existe $\sigma_{1}>0$ tal que

$$
\frac{1}{4}\|u\|_{\lambda}^{2} \leqslant \Phi_{\lambda}^{\prime}(u)(u) \text { para }\|u\|_{\lambda}<\sigma_{1}
$$

Além disso, se $c<\sigma_{1}^{2} \frac{q-2}{2 q}$ e $\left(u_{n}\right)$ é $(P S)_{c}$ para $\Phi_{\lambda}$, então

$$
\limsup _{n \rightarrow \infty}\left\|u_{n}\right\|_{\lambda}^{2} \leqslant \frac{2 q c}{q-2}<\sigma_{1}^{2}
$$

Assim, $\left\|u_{n}\right\|_{\lambda}<\sigma_{1}$ para $n$ grande. Então por (2.3),

$$
\frac{1}{4}\left\|u_{n}\right\|_{\lambda}^{2} \leqslant \Phi_{\lambda}^{\prime}\left(u_{n}\right)\left(u_{n}\right)=o(1)\left\|u_{n}\right\|_{\lambda}
$$

o que implica $\left\|u_{n}\right\|_{\lambda} \rightarrow 0$ quando $n \rightarrow+\infty$. Portanto $\Phi\left(u_{n}\right) \rightarrow 0$, isto é, $c=0$. Logo, $c_{0}=\frac{\sigma_{1}^{2}(q-2)}{2 q}$.

A demonstração do próximo lema pode ser encontrada também em [BW95].

Lema 2.1.4. Existe $\delta_{0}>0$ tal que para qualquer sequência $\left(u_{n}\right)$ que é $(P S)_{c}$ de $\Phi_{\lambda}$ com $\lambda>0$ e c $>0$, temos

$$
\liminf _{n \rightarrow+\infty}\left\|u_{n}\right\|_{L^{s+1}\left(\mathbb{R}^{N}\right)}^{s+1} \geqslant \delta_{0} c
$$

Demonstração: do Lema 2.1.3 sabemos

$$
\left\|u_{n}\right\|_{\lambda}^{2} \leqslant \frac{2 q}{q-2} c+o(1) \text { quando } n \rightarrow+\infty .
$$

Agora, do Lema 2.1.2, da hipótese (A3) e da hipótese (A4), para todo $\epsilon>0$, existe $A(\epsilon)>0$ tal que $f(x, u) u \leqslant \epsilon|u|^{2}+A(\epsilon)|u|^{s+1}$, ou seja,

$$
\frac{1}{2} f(x, u) u-F(x, u) \leqslant \epsilon|u|^{2}+A(\epsilon)|u|^{s+1}
$$


para todo $x \in \mathbb{R}^{N}$ e $u \in \mathbb{R}$. Usando que $\left(u_{n}\right)$ é uma sequência $(P S)_{c}$ e $(2.1)$,

$$
\begin{aligned}
c & =\lim _{n \rightarrow+\infty}\left(\Phi_{\lambda}\left(u_{n}\right)-\frac{1}{2} \Phi_{\lambda}^{\prime}\left(u_{n}\right)\left(u_{n}\right)\right) \\
& =\lim _{n \rightarrow+\infty} \int_{\mathbb{R}^{N}}\left(\frac{1}{2} f\left(x, u_{n}\right) u_{n}-F\left(x, u_{n}\right)\right) d x \\
& \leqslant \liminf _{n \rightarrow+\infty} \int_{\mathbb{R}^{N}}\left(\epsilon\left|u_{n}\right|^{2}+A(\epsilon)\left|u_{n}\right|^{s+1}\right) d x \\
& \leqslant \liminf _{n \rightarrow+\infty}\left(\frac{2 q \epsilon}{q-2} c+A(\epsilon)\left\|u_{n}\right\|_{L^{s+1}\left(\mathbb{R}^{N}\right)}^{s+1}\right) .
\end{aligned}
$$

Escolhendo $\epsilon=\frac{q-2}{4 q}$ temos

$$
\liminf _{n \rightarrow+\infty}\left\|u_{n}\right\|_{L^{s+1}\left(\mathbb{R}^{N}\right)}^{s+1} \geqslant \frac{1}{2 A(\epsilon)} c .
$$

Tomando $\delta_{0}:=\frac{1}{2 A(\epsilon)}$, temos

$$
\liminf _{n \rightarrow+\infty}\left\|u_{n}\right\|_{L^{s+1}\left(\mathbb{R}^{N}\right)}^{s+1} \geqslant \delta_{0} c
$$

Lema 2.1.5. Seja $C_{1}>0$ uma constante fixada. Então para todo $\epsilon>0$, existem $\Lambda_{\epsilon}>0$ e $R_{\epsilon}>0$ tal que se $\left(u_{n}\right)$ é uma sequência $(P S)_{c}$ de $\Phi_{\lambda}$ com $c \leqslant C_{1}$ e $\lambda \geqslant \Lambda_{\epsilon}$, então

$$
\limsup _{n \rightarrow \infty}\left\|u_{n}\right\|_{L^{s+1}\left(B_{R_{\epsilon}}^{c}\right)}^{s+1} \leqslant \epsilon
$$

onde,

$$
B_{R_{\epsilon}}^{c}=\left\{x \in \mathbb{R}^{N}:|x|>R_{\epsilon}\right\}
$$

Demonstração: para $R>0$ dado, consideremos os seguintes conjuntos:

$$
\begin{aligned}
& A_{1}(R)=\left\{x \in \mathbb{R}^{N}:|x|>R, a(x) \geqslant M_{0}\right\}, \\
& A_{2}(R)=\left\{x \in \mathbb{R}^{N}:|x|>R, a(x) \leqslant M_{0}\right\},
\end{aligned}
$$

onde $M_{0}$ é dado por $\left(A_{2}\right)$. Usando a desigualdade de interpolação de Hölder para $2<s+1<2^{*}$,

$$
\left|u_{n}\right|_{L^{s+1}\left(B_{R_{\epsilon}}^{c}\right)} \leqslant\left|u_{n}\right|_{L^{2}\left(B_{R_{\epsilon}}^{c}\right)}^{1-\theta}\left|u_{n}\right|_{L^{2^{*}}\left(B_{R_{\epsilon}}^{c}\right.}^{\theta} .
$$

Pela desigualdade de Sobolev-Gagliardo-Nirenberg,

$$
\left|u_{n}\right|_{L^{2^{*}\left(B_{R}^{c}\right)}} \leqslant C\left|\nabla u_{n}\right|_{L^{2}\left(B_{R_{\epsilon}}^{c}\right)} .
$$


Logo,

$$
\int_{B_{R_{\epsilon}}^{c}}\left|u_{n}\right|^{s+1} d x \leqslant C\left|u_{n}\right|_{L^{2}\left(B_{R_{\epsilon}}^{c}\right)}^{1-\theta}\left|\nabla u_{n}\right|_{L^{2}\left(B_{R_{\epsilon}}^{c}\right)}^{\theta} \cdot
$$

Escolhendo $\theta=\frac{N(s-1)}{2(s+1)}$ e usando o Lema 2.1.3 resulta que a menos de subsequência temos

$$
\int_{B_{R \epsilon}^{c}}\left|u_{n}\right|^{s+1} d x \leqslant C\left(\frac{2 q}{q-2} C_{1}\right)^{\theta(s+1)}\left(\int_{A_{1}(R \epsilon)} u_{n}^{2} d x+\int_{A_{2}(R \epsilon)} u_{n}^{2} d x\right)^{\frac{(1-\theta)(s+1)}{2}} .
$$

Analisaremos agora as integrais $\int_{A_{1}(R \epsilon)} u_{n}^{2} d x$ e $\int_{A_{2}(R \epsilon)} u_{n}^{2} d x$.

Do Lema 2.1.3.

$$
\begin{aligned}
\int_{A_{1}(R \epsilon)} u_{n}^{2} d x & \leqslant \frac{1}{\lambda M_{0}+1} \int_{\mathbb{R}^{N}}(\lambda a(x)+1) u_{n}^{2} d x \\
& \leqslant \frac{1}{\lambda M_{0}+1}\left\|u_{n}\right\|_{\lambda}^{2} \leqslant \frac{1}{\lambda M_{0}+1}\left(\frac{2 q C_{1}}{q-2}+o(1)\right) \text {, quando } n \rightarrow+\infty .
\end{aligned}
$$

Novamente pelo Lema 2.1.3 e usando a desigualdade de Hölder,

$$
\begin{aligned}
\int_{A_{2}(R \epsilon)} u_{n}^{2} d x & \leqslant\left[\int_{\mathbb{R}^{N}}\left|u_{n}\right|^{2 r} d x\right]^{\frac{1}{r}}\left[\int_{A_{2}(R \epsilon)} d x\right]^{\frac{1}{r^{\prime}}} \\
& \leqslant C\left\|u_{n}\right\|_{H^{1}\left(\mathbb{R}^{N}\right)}^{2} \mu\left(A_{2}(R)\right)^{\frac{1}{r^{\prime}}} \\
& \leqslant C\left\|u_{n}\right\|_{\lambda}^{2} \mu\left(A_{2}(R \epsilon)\right)^{\frac{1}{r^{\prime}}} \\
& \leqslant C \frac{2 q C_{1}}{q-2} \mu\left(A_{2}(R \epsilon)\right)^{\frac{1}{r^{\prime}}}
\end{aligned}
$$

para $1<r<\frac{N}{N-2}$ e $r^{\prime}=\frac{r}{r-1}$, onde $C=C(N, r)$ é uma constante positiva. Como $\int_{A_{1}(R \epsilon)} u_{n}^{2} d x \mathrm{e} \int_{A_{2}(R \epsilon)} u_{n}^{2} d x$ podem ser tão pequenos quanto se queira desde que $A_{1}(R \epsilon), \lambda \mathrm{e}$ $R \epsilon$ sejam respectivamente suficientemente grandes, então desde que $\lambda$ seja suficientemente grande

$$
\int_{B_{R_{\epsilon}}^{c}}\left|u_{n}\right|^{s+1} d x \rightarrow 0, \quad \text { quando } n \rightarrow \infty
$$

$\operatorname{Logo}, \limsup _{n \rightarrow+\infty}\left\|u_{n}\right\|_{L^{s+1}\left(B_{R_{\epsilon}}^{c}\right)}^{s+1}<\epsilon$.

Lema 2.1.6. Seja $\lambda \geqslant 0$ fixado e seja $\left(u_{n}\right)$ uma sequência $(P S)_{c}$ de $\Phi_{\lambda}$. Então, a menos de subsequência, $u_{n} \rightarrow u$ em $E$, com u sendo uma solução fraca de $\left(S_{\lambda u}\right)$. Além disso, $v_{n}=u_{n}-u$ é uma sequência $(P S)_{c^{\prime}}$, com $c^{\prime}=c-\Phi_{\lambda}(u)$.

Demonstração: primeiramente observe que pelo Lema 2.3, $\left(u_{n}\right)$ é uma sequência limitada em $E$. Então, a menos de subsequência, $u_{n} \rightarrow u$ fracamente em $E$, quando 
$n \rightarrow+\infty$. Vamos mostrar que $u$ é um ponto crítico de $\Phi_{\lambda}$. Como $u_{n}$ é uma sequência limitada e converge fraco para $u$ temos:

$$
u_{n} \rightarrow \text { u em } L_{L o c}^{p}\left(\mathbb{R}^{N}\right) \text {, para todo } 2 \leqslant p<2^{*} .
$$

Portanto, para todo $w \in E$ temos

$$
\Phi_{\lambda}^{\prime}(u)(w)=\lim _{n \rightarrow+\infty} \Phi_{\lambda}^{\prime}\left(u_{n}\right)(w)=0
$$

pois $\left(u_{n}\right)$ é uma sequência $(P S)_{c}$. Portanto $u$ é um ponto crítico de $\Phi_{\lambda}$, ou seja, $u$ é uma solução fraca de $\left(S_{\lambda}\right)$. Vamos agora mostrar que $v_{n}=u_{n}-u$ é uma sequência $(P S)_{c^{\prime}}$, $\operatorname{com} c^{\prime}=c-\Phi_{\lambda}(u)$, isto é,

- $\Phi_{\lambda}\left(v_{n}\right) \rightarrow c-\Phi_{\lambda}(u), n \rightarrow+\infty$

- $\Phi_{\lambda}^{\prime}\left(v_{n}\right) \rightarrow 0, n \rightarrow+\infty$

Primeiramente, vamos mostrar que, $\Phi_{\lambda}\left(v_{n}\right) \rightarrow c-\Phi_{\lambda}(u)$, quando $n \rightarrow+\infty$. Como

$$
\begin{aligned}
\Phi_{\lambda}\left(v_{n}\right) & =\Phi_{\lambda}\left(u_{n}\right)-\Phi_{\lambda}(u)-\left\langle v_{n}, u\right\rangle_{E} \\
& +\int_{\mathbb{R}^{N}}\left[F\left(x, v_{n}+u\right)-F\left(x, v_{n}\right)-F(x, u)\right] d x
\end{aligned}
$$

e $\left\langle v_{n}, u\right\rangle_{E} \rightarrow 0$, quando $n \rightarrow+\infty$, então basta mostrar que

$$
\int_{\mathbb{R}^{N}}\left[F\left(x, v_{n}+u\right)-F\left(x, v_{n}\right)-F(x, u)\right] d x \rightarrow 0 \text {, quando } n \rightarrow+\infty .
$$

Dado $\epsilon>0$, escolha $R(\epsilon)>0$ tal que

$$
\int_{B_{R(\epsilon)}^{c}}|u|^{s+1} d x \leqslant \epsilon \text { e } \int_{B_{R(\epsilon)}^{c}}|u|^{2} d x \leqslant \epsilon,
$$

onde $B_{R(\epsilon)}^{c}=\left\{x \in \mathbb{R}^{N}:|x| \geqslant R(\epsilon)\right\}$. Daí,

$$
\begin{aligned}
\int_{B_{R(\epsilon)}^{c}}\left|F\left(v_{n}+u\right)\right|-\mid & F\left(v_{n}\right)\left|d x \leqslant \int_{B_{R(\epsilon)}^{c}}\right| f\left(v_{n}+\xi(x, n) u\right) \| u \mid d x \\
& \leqslant C \int_{B_{R(\epsilon)}^{c}}\left(\left|v_{n}\right|+|u|+\left(\left|v_{n}\right|+|u|\right)^{s}\right)|u| d x \\
& \leqslant C\left\|v_{n}\right\|_{L^{2}\left(B_{R(\epsilon)}^{c}\right)} \mid\|u\|_{L^{2}\left(B_{R(\epsilon)}^{c}\right)}+C\|u\|_{L^{2}\left(B_{R(\epsilon)}^{c}\right)}^{2} \\
& +C\left[\int_{B_{R(\epsilon)}^{c}}\left(\left|v_{n}\right|+|u|\right)^{s+1} d x\right]^{\frac{s}{s+1}}\left[\int_{B_{R(\epsilon)}^{c}}|u|^{s+1} d x\right]^{\frac{1}{s+1}} \\
& =o\left(\epsilon^{\left.\frac{1}{s+1}\right)},\right.
\end{aligned}
$$


quando $\epsilon \rightarrow 0$ uniformemente em $n$, com $\mathrm{C}$ sendo independente de $n$. Por outro lado, usando a compacidade das imersões de Sobolev temos

$$
\int_{B_{R_{\epsilon}}}\left|F\left(x, v_{n}+u\right)-F\left(x, v_{n}\right)-F(x, u)\right| d x \rightarrow 0, \text { quando } n \rightarrow+\infty .
$$

Usando 2.5 e 2.8), segue que

$$
\Phi_{\lambda}\left(v_{n}\right) \rightarrow c-\Phi_{\lambda}(u)
$$

Por fim, vamos mostrar que $\Phi_{\lambda}^{\prime}\left(v_{n}\right) \rightarrow 0$. Seja $w \in E$, então de 2.6 temos

$$
\Phi_{\lambda}^{\prime}\left(v_{n}\right)(w)=\Phi_{\lambda}^{\prime}\left(u_{n}\right)(w)-\int_{\mathbb{R}^{N}}\left[\left(f\left(x, v_{n}\right)+f\left(x, u_{n}\right)+f(x, u)\right) w\right] d x .
$$

Como $\Phi_{\lambda}^{\prime}\left(u_{n}\right) \rightarrow 0$ temos que mostrar que

$$
\int_{\mathbb{R}^{n}}\left(f\left(x, v_{n}\right)-f\left(x, u_{n}\right)+f(x, u)\right) w d x \rightarrow 0, \text { quando } n \rightarrow+\infty .
$$

Dado $\epsilon>0$, escolha $R(\epsilon)>0$, como em (2.7), para obter

$$
\int_{B_{R(\epsilon)}^{c}}|f(x, u) w| d x \leqslant C \int_{B_{R(\epsilon)}^{c}}\left(|u|+|u|^{s}\right)|w| d x \leqslant C \sqrt{\epsilon}\|w\|_{\lambda}+C \epsilon^{\frac{1}{s+1}}\|w\|_{\lambda} .
$$

Além disso, dado $x \in B_{R(\epsilon)}^{c}$, para algum $0 \leq \xi(x) \leq 1$,

$$
\begin{aligned}
\int_{B_{R(\epsilon)}^{c}}\left|f\left(x, v_{n}\right)-f\left(x, v_{n}+u\right)\right||w| d x & \leqslant \int_{B_{R(\epsilon)}^{c}}\left|f^{\prime}\left(x, v_{n}+\xi u\right)\|u\| w\right| d x \\
& \leqslant C \int_{B_{R(\epsilon)}^{c}}\left(1+\left(\left|v_{n}\right|+|u|^{s-1}\right)\right)|u \| w| d x \\
& \leqslant C\|u\|_{L^{2}\left(B_{R(\epsilon)}^{c}\right)}\|w\|_{\lambda}+C\|w\|_{\lambda}\|u\|_{L^{s+1}\left(B_{R(\epsilon)}^{c}\right)} \\
& =o(\sqrt{\epsilon})+o\left(\epsilon^{\frac{1}{s+1}}\right)\|w\|_{\lambda} .
\end{aligned}
$$

Usando 2.5 temos $\Phi_{\lambda}^{\prime}\left(v_{n}\right) \rightarrow 0$. Logo, $v_{n}=u_{n}-u$ é uma sequência $(P S)_{c^{\prime}}$ de $\Phi_{\lambda}$, onde $c^{\prime}=c-\Phi \lambda(u)$.

Agora, depois de provarmos esses lemas podemos finalmente demonstrar o principal resultado desta seção.

Demonstração da Proposição 2.1.1: fixe $0<\epsilon<\frac{\delta_{0} c_{0}}{2}$, onde $c_{0}>0$ é dado pelo Lema 2.1.3 e $\delta_{0}$ pelo Lema 2.1.4. Então dado $C_{0}>0$, escolha $\Lambda_{\epsilon}>0$ e $R_{\epsilon}>0$ como no Lema 2.1.5. Tome $\Lambda_{0}=\Lambda_{\epsilon}$. Considere $\left(u_{n}\right)$ uma sequência $(P S)_{c}$ de $\Phi_{\lambda}$, com $\lambda \geqslant \Lambda_{0}$ e $c \leqslant C_{0}$. Pelo Lema 2.1.6, $v_{n}=u_{n}-u$ é uma sequência $(P S)_{c^{\prime}}$ de $\Phi_{\lambda}$, com $c^{\prime}=c-\Phi_{\lambda}(u)$. 
Se $c^{\prime}>0$, então pelo Lema 2.1.3 temos $c^{\prime} \geqslant c_{0}>0$. Do Lema 2.1.4. $\left(v_{n}\right)$ satisfaz

$$
\liminf _{n \rightarrow+\infty}\left\|v_{n}\right\|_{L^{s+1}\left(\mathbb{R}^{N}\right)}^{s+1} \geqslant \delta_{0} c^{\prime} \geqslant \delta_{0} c_{0}
$$

Observe que

$$
\begin{aligned}
\delta_{0} c_{0} \leqslant \liminf _{n \rightarrow+\infty}\left\|v_{n}\right\|_{L^{s+1}\left(\mathbb{R}^{N}\right)}^{s+1} & =\liminf _{n \rightarrow+\infty}\left\|v_{n}\right\|_{L^{s+1}\left(B_{R_{\epsilon}}\right)}^{s+1}+\liminf _{n \rightarrow+\infty}\left\|v_{n}\right\|_{L^{s+1}\left(B_{R_{\epsilon}}^{c}\right)}^{s+1} \\
& =\liminf _{n \rightarrow+\infty}\left\|v_{n}\right\|_{L^{s+1}\left(B_{R_{\epsilon}}^{c}\right)}^{s+1} \leqslant \limsup _{n \rightarrow+\infty}\left\|v_{n}\right\|_{L^{s+1}\left(B_{R_{\epsilon}}^{c}\right)}^{s+1}
\end{aligned}
$$

pois $\liminf _{n \rightarrow+\infty}\left\|v_{n}\right\|_{L^{s+1}\left(B_{R_{\epsilon}}\right)}^{s+1}=0$. Usando o Lema 2.1 .5 temos

$$
\limsup _{n \rightarrow+\infty}\left\|v_{n}\right\|_{L^{s+1}\left(B_{R_{\epsilon}}^{c}\right)}^{s+1} \leqslant \epsilon<\frac{\delta_{0} c_{0}}{2}
$$

Logo, de 2.9 e 2.10 temos

$$
\delta_{0} c_{0}<\frac{\delta_{0} c_{0}}{2}
$$

O que é uma contradição. Portanto $c^{\prime}=0$ e daí, $v_{n} \rightarrow 0$, ou seja, $u_{n} \rightarrow u$ e $\Phi_{\lambda}(u)=c$. Logo, $\Phi_{\lambda}$ satisfaz a condição $(P S)_{c}$ para todo $c \leqslant C_{0}$.

\subsection{Prova dos Resultados Principais}

Nesta seção demonstraremos os principais resultados deste trabalho. Primeiramente dado $\Omega \subset \mathbb{R}^{N}$ um conjunto limitado com fronteira suave, consideremos o problema de Dirichlet,

$$
-\Delta u+u=u^{p}, \quad u>0 \text { em } \Omega, \quad u=0 \text { sobre } \partial \Omega,
$$

e a variedade de Nehari correspondente,

$$
N(p, \Omega)=\left\{u \in H_{0}^{1}(\Omega) \backslash\{0\}: \int_{\Omega}\left(|\nabla u|^{2}+u^{2}\right) d x=\int_{\Omega}|u|^{p+1} d x\right\} .
$$

Considere também

$$
c(p, \Omega)=\inf _{u \in N(p, \Omega)} \Phi_{p, \Omega}(u)
$$

onde

$$
\Phi_{p, \Omega}(u)=\frac{1}{2} \int_{\Omega}\left(|\nabla u|^{2}+u^{2}\right) d x-\frac{1}{p+1} \int_{\Omega}|u|^{p+1} d x, \text { para } u \in H_{0}^{1}(\Omega)
$$

e

$$
m(p, \lambda, \Omega)=\inf _{u \in V_{p}} \int_{\Omega}\left(|\nabla u|^{2}+\lambda u^{2}\right) d x
$$

onde

$$
V_{p}=V_{p}(\Omega) \doteq\left\{u \in H_{0}^{1}(\Omega):\|u\|_{L^{p+1}}(\Omega)=1\right\}
$$


Pela Proposição A.0.15 do Apêndice A, o funcional $\Phi_{p, \Omega}$ está bem definido em $H_{0}^{1}(\Omega)$ e é de classe $C^{1}$. Além disso, para $r>0$ fixemos $B_{r}=\left\{x \in \mathbb{R}^{N}:|x|<r\right\}$ e definimos

$$
c(p, r):=c\left(p, B_{r}\right)
$$

Para $u \in N(p, \Omega)$ vamos definir o centro de massa de $u$.

Definição 2.2.1. Seja $u \in N(p, \Omega)$. O centro de massa de u é dada por:

$$
\beta(u)=\frac{\int_{\Omega}|u|^{p+1} x d x}{\int_{\Omega}|u|^{p+1} d x} .
$$

Novamente, a fim de demonstrarmos os principais resultados deste trabalho, provaremos alguns lemas.

Lema 2.2.2. $c(p, \Omega) \rightarrow \frac{1}{N} S^{\frac{N}{2}}$, quando $p \rightarrow 2^{*}-1$, onde $S \doteq m\left(2^{*}, \lambda, \Omega\right)$.

Para provarmos o lema acima, demonstraremos duas proposições.

Proposição 2.2.3. $c(p, \Omega)=\frac{p-1}{2(p+1)}(m(p, \lambda, \Omega))^{\frac{p+1}{p-1}}$.

Demonstração: a prova será feita em dois passos (i) a (ii) a seguir:

(i) $c(p, \Omega) \leqslant \frac{p-1}{2(p+1)}(m(p, \lambda, \Omega))^{\frac{p+1}{p-1}}$.

Seja $u \in V_{p}$, assim

$$
\Phi_{p, \Omega}(t u)=\frac{t^{2}}{2}\|u\|^{2}-\frac{t^{p+1}}{p+1}\|u\|_{L^{p+1}(\Omega)} .
$$

Considere $\bar{t} \in \mathbb{R}$ tal que $\bar{t} u \in N(p, \Omega)$, daí

$$
\bar{t}\|u\|^{2}-\bar{t}^{p}\|u\|_{L^{p+1}(\Omega)}^{p+1}=0,
$$

implicando

$$
\bar{t}=\left(\frac{\|u\|^{2}}{\|u\|_{L^{p+1}(\Omega)}^{p+1}}\right)^{\frac{1}{p-1}}=\|u\|^{\frac{2}{p-1}}, \quad \text { pois }\|u\|_{L^{p+1}(\Omega)}^{p+1}=1 .
$$

Logo,

$$
\Phi_{p, \Omega}\left(\|u\|^{\frac{2}{p-1}} u\right)=\frac{p-1}{2(p+1)}\|\| u\left\|^{\frac{2}{p-1}} u\right\|^{2}=\frac{p-1}{2(p+1)}\|u\|^{\frac{2(p+1)}{p-1}} .
$$

Como $c(p, \Omega)=\inf _{u \in N(p, \Omega)} \Phi_{p, \Omega}(u)$, então

$$
\left(\frac{2(p+1)}{p-1} c(p, \Omega)\right)^{\frac{p-1}{p+1}} \leqslant\left(\frac{2(p+1)}{p-1} \Phi_{p, \Omega}\left(\|u\|^{\frac{2}{p-1}} u\right)\right)^{\frac{p-1}{p+1}}
$$


Portanto,

$$
\left(\frac{2(p+1)}{p-1} c(p, \Omega)\right)^{\frac{p-1}{p+1}} \leqslant\|u\|^{2}, \text { para todo } u \in V_{p}
$$

Assim,

$$
\left(\frac{2(p+1)}{p-1} c(p, \Omega)\right)^{\frac{p-1}{p+1}} \leqslant m(p, \lambda, \Omega)
$$

isto é,

$$
c(p, \Omega) \leqslant \frac{p-1}{2(p+1)} m(p, \lambda, \Omega)^{\frac{p+1}{p-1}}
$$

(ii) $c(p, \Omega) \geqslant \frac{p-1}{2(p+1)} m(p, \lambda, \Omega)^{\frac{p+1}{p-1}}$.

Seja $u \in N(p, \Omega)$. Observe que para $u \in N(p, \Omega)$, temos $\frac{u}{\|u\|_{L^{p+1}(\Omega)}} \in V_{p}$. Assim,

$$
\left\|\frac{u}{\|u\|_{L^{p+1}(\Omega)}}\right\|=\frac{\|u\|}{\|u\|_{L^{p+1}(\Omega)}}=\frac{\|u\|}{\|u\|^{\frac{2}{p+1}}}=\|u\|^{1-\frac{2}{p+1}}=\|u\|^{\frac{p-1}{p+1}},
$$

uma vez que $\|u\|^{2}=\|u\|_{L^{p+1}(\Omega)}^{p+1}$. Portanto,

$$
\left\|\frac{u}{\|u\|_{L^{p+1}(\Omega)}}\right\|^{2}=\|u\|^{\frac{2(P+1)}{P-1}} .
$$

Assim,

$$
m(p, \lambda, \Omega) \leqslant\left\|\frac{u}{\|u\|_{L^{p+1}}(\Omega)}\right\|^{2}=\|u\|^{\frac{2(p-1)}{p+1}}=\left(\frac{2(p+1)}{p-1} \Phi_{p, \Omega}(u)\right)^{\frac{p-1}{p+1}}
$$

pois, novamente como $u \in N(p, \Omega),\|u\|^{2}=\frac{2(p+1)}{p-1} \Phi_{p, \Omega}(u)$. Logo,

$$
\frac{p-1}{2(p+1)} m(p, \lambda, \Omega)^{\frac{p+1}{p-1}} \leqslant \Phi_{p, \Omega}(u),
$$

para todo $u \in N(p, \Omega)$. Portanto,

$$
\frac{p-1}{2(p+1)} m(p, \lambda, \Omega)^{\frac{p+1}{p-1}} \leqslant c(p, \Omega) .
$$

Deste modo, de (i) e (ii) segue que

$$
c(p, \Omega)=\frac{p-1}{2(p+1)} m(p, \Omega)^{\frac{p+1}{p-1}} .
$$

Proposição 2.2.4. Para qualquer domínio limitado $\Omega \subset \mathbb{R}^{N}$ e para qualquer $\lambda \geqslant 0$,

$$
\lim _{p \rightarrow 2^{*}} m(p, \lambda, \Omega)=S
$$


Demonstração: primeiramente usando a desigualdade de Hölder,

$$
\|u\|_{L^{p}(\Omega)}=\left[\int_{\Omega}|u|^{p} d x\right]^{\frac{1}{p}} \leqslant\|u\|_{L^{q}(\Omega)}|\Omega|^{\frac{q-p}{q p}}
$$

para todo $p, q \in\left[2,2^{*}\right]$ com $p<q$ e para todo $u \in H_{0}^{1}(\Omega)$.

Deste modo,

implicando

$$
\frac{\int_{\Omega}\left(|\nabla u|^{2}+\lambda u^{2}\right) d x}{\|u\|_{L^{p}(\Omega)}^{2}} \geqslant|\Omega|^{\frac{-2(q-p)}{q p}} \frac{\int_{\Omega}\left(|\nabla u|^{2}+\lambda u^{2}\right) d x}{\|u\|_{L^{q}(\Omega)}^{2}},
$$

$$
\begin{aligned}
& m(p, \lambda, \Omega) \geqslant|\Omega|^{\frac{-2\left(2^{*}-p\right)}{2^{*} p}} S, \\
& m(p, \lambda, \Omega) \leqslant|\Omega|^{\frac{p-2}{p}} m(2, \lambda, \Omega) .
\end{aligned}
$$

Portanto para qualquer $\lambda \geqslant 0$ e $\Omega, m(p, \lambda, \Omega)$ é limitado superiormente e inferiormente quando $p \in\left[2,2^{*}\right]$. Defina

$$
m=\liminf _{p \rightarrow 2^{*}} m(p, \lambda, \Omega) \quad \text { e } \quad M=\limsup _{p \rightarrow 2^{*}} m(p, \lambda, \Omega) .
$$

Para concluir a demonstração basta mostrarmos que

$$
m=S=M
$$

Mostraremos primeiro que $m=S$.

De (2.12) segue que $m \geqslant S$. Suponha $m>S$. Portanto podemos escolher $\epsilon \in(0, m-S)$ e escolher uma função $\bar{u} \in H_{0}^{1}(\Omega)$ tal que

$$
\frac{\int_{\Omega}\left(|\nabla \bar{u}|^{2}+\lambda \bar{u}^{2}\right) d x}{\|\bar{u}\|_{L^{2^{*}}(\Omega)}^{2}}<S+\frac{\epsilon}{2}
$$

Por outro lado, pela continuidade da aplicação $p \mapsto\|\bar{u}\|_{L^{p}(\Omega)}$, existe $\bar{p} \in\left(2,2^{*}\right)$ tal que para todo $p \in\left[\bar{p}, 2^{*}\right)$ temos

$$
\left|\frac{\int_{\Omega}\left(|\nabla \bar{u}|^{2}+\lambda \bar{u}^{2}\right) d x}{\|\bar{u}\|_{L^{p}(\Omega)}^{2}}-\frac{\int_{\Omega}\left(|\nabla \bar{u}|^{2}+\lambda \bar{u}^{2}\right) d x}{\|\bar{u}\|_{L^{2^{*}}(\Omega)}^{2}}\right|<\frac{\epsilon}{2}
$$

Logo, para todo $p \in\left[\bar{p}, 2^{*}\right]$ temos

$$
m(p, \lambda, \Omega) \leqslant \frac{\int_{\Omega}\left(|\nabla \bar{u}|^{2}+\lambda \bar{u}^{2}\right) d x}{\|\bar{u}\|_{L^{p}(\Omega)}^{2}}<\frac{\int_{\Omega}\left(|\nabla \bar{u}|^{2}+\lambda \bar{u}^{2}\right) d x}{\|\bar{u}\|_{L^{2^{*}}(\Omega)}^{2}}+\frac{\epsilon}{2}<S+\epsilon<m,
$$


o que é um absurdo, pois $m=\liminf _{p \rightarrow 2^{*}} m(p, \lambda, \Omega)$. Portanto, $m=S$.

Por outro lado, usando novamente que

$$
m(p, \lambda, \Omega)<\frac{\int_{\Omega}\left(|\nabla \bar{u}|^{2}+\lambda \bar{u}^{2}\right) d x}{\|\bar{u}\|_{L^{p}(\Omega)}^{2}}<S+\epsilon
$$

para todo $p \in\left[\bar{p}, 2^{*}\right)$, temos

$$
M=\limsup _{p \rightarrow 2^{*}} m(p, \lambda, \Omega) \leqslant S+\epsilon
$$

para todo $\epsilon>0$. Portanto, $M \leqslant S=m=S$, isto é, $M=S=m$.

Finalmente com esses dois resultados, a demonstração do Lema 2.2 .2 é trivial, na verdade a prova é exatamente a demonstração das duas proposições anteriores.

No que segue, denotemos $S_{2}$ a melhor constante de Sobolev da imersã ${ }^{1} D^{1,2}\left(\mathbb{R}^{N}\right) \subset$ $L^{2^{*}}\left(\mathbb{R}^{N}\right)$, isto é,

$$
S_{2}=\inf \left\{\int_{\mathbb{R}^{N}}|\nabla u|^{2} d x: u \in D^{1,2}\left(\mathbb{R}^{N}\right) \text { e }\|u\|_{L^{2^{*}\left(\mathbb{R}^{N}\right)}}=1\right\} .
$$

De agora em diante, admitiremos que $\Omega$ é o interior de $a^{-1}(0)$. Pela hipótese (A1), existe $r>0$ tal que as inclusões $\Omega^{-} \hookrightarrow \Omega \hookrightarrow \Omega^{+}$são homotopicamente equivalentes, onde

$$
\Omega^{+}=\left\{x \in \mathbb{R}^{N}: \operatorname{dist}(x, \Omega) \leqslant r\right\}
$$

e

$$
\Omega^{-}=\{x \in \Omega: \operatorname{dist}(x, \partial \Omega) \geqslant r\} .
$$

A demonstração do lema a seguir foi extraída do trabalho [BC91] e faz uso do lema de concentração e compacidade (veja Apêndice B).

Proposição 2.2.5. Se $\left(u_{n}\right) \subset V_{p}(\Omega)$ é tal que $\left\|u_{n}\right\|^{2} \rightarrow S_{2}$, então $\operatorname{dist}\left(\beta\left(u_{n}\right), \Omega\right) \rightarrow 0$.

Demonstração: suponha por absurdo que $\operatorname{dist}\left(\beta\left(u_{n}\right), \Omega\right) \nrightarrow 0$. Assim, a menos de subsequência, podemos admitir que

$$
\operatorname{dist}\left(\beta\left(u_{n}\right), \Omega\right)>r>0
$$

e

$$
\left\{\begin{array}{lc}
u_{n} \rightarrow u & \text { em } D^{1,2}\left(\mathbb{R}^{N}\right) \\
\left|\nabla\left(u_{n}-u\right)\right|^{2} \rightarrow \mu & \text { em } M\left(\mathbb{R}^{N}\right) \\
\left|u_{n}-u\right|^{2^{*}} \rightarrow \nu & \text { em } M\left(\mathbb{R}^{N}\right) \\
u_{n} \rightarrow u & \text { qtp em } \Omega
\end{array}\right.
$$

${ }^{1}$ Veja a definição do espaço $D^{1,2}\left(\mathbb{R}^{N}\right)$ no Apêndice B. 
onde $\mu$ e $\nu$ são medidas finitas e $M\left(\mathbb{R}^{N}\right)$ denota o espaço das medidas finitas em $\mathbb{R}^{N}$.

Pela Proposição B.0.21 do Apêndice B resulta que

$$
S_{2}=\|\nabla u\|_{L^{2}(\Omega)}^{2}+\|\mu\|+\mu_{\infty}
$$

$\mathrm{e}$

$$
1=\|u\|_{L^{2^{*}(\Omega)}}^{2^{*}}+\|\nu\|+\nu_{\infty}
$$

onde $\mu_{\infty}=\nu_{\infty}=0$, pois $\Omega$ é limitado. Assim,

$$
\|\nu\|^{\frac{2}{2^{*}}} \leqslant S_{2}^{-1}\|\mu\|
$$

e

$$
\|u\|_{L^{2^{*}}(\Omega)}^{2} \leqslant S_{2}^{-1}\|\nabla u\|_{L^{2}(\Omega)}^{2} .
$$

Usando as equações dadas acima temos

$$
S_{2}=\|\nabla u\|_{L^{2}(\Omega)}^{2}+\|\mu\| \geqslant S_{2}\left(\|u\|_{L^{2^{*}}(\Omega)}^{2}+\|\nu\|^{\frac{2}{2^{*}}}\right) .
$$

Implicando que,

$$
\|u\|_{L^{2^{*}}(\Omega)}^{2^{*}}+\|\nu\|=1 \geqslant\|u\|_{L^{2^{*}(\Omega)}}^{2}+\|\nu\|^{\frac{2}{2^{*}}}
$$

Por 2.15), $\|\nu\| \in[0,1]$. Se $\|\nu\| \in(0,1)$, então

$$
\|\nu\|^{\frac{2}{2^{*}}}>\|\nu\|
$$

De 2.17$)$ e 2.18$)$ segue que

$$
\|u\|_{L^{2^{*}(\Omega)}}^{2^{*}}+\|\nu\|>\|u\|_{L^{2^{*}(\Omega)}}^{2}+\|\nu\|
$$

Mas isto é impossível pois, por $2.15,\|u\|_{L^{2^{*}(\Omega)}}^{2^{*}} \in[0,1]$ e $2^{*}>2$. Logo, $\|\nu\|=0$ ou $\|\nu\|=1$. Se $\|\nu\|=0$, então, por (2.15),

$$
\|u\|_{L^{2^{*}(\Omega)}}=1
$$

Usando 2.14 temos

$$
S_{2}=\|\nabla u\|_{L^{2}(\Omega)}^{2}+\|\mu\| \geqslant\|\nabla u\|_{L^{2}(\Omega)}^{2} .
$$

Combinando 2.16, 2.19 e 2.20

$$
S_{2} \leqslant\|\nabla u\|_{L^{2}(\Omega)}^{2} \leqslant S_{2} .
$$


Logo, $S_{2}$ é atingido por uma função $u \in L^{2^{*}}(\Omega)$, o que contradiz a Proposição B.0.22 do Apêndice B. Portanto, $\|\nu\|=1$, ou seja, $u=0$. Assim, do Lema B.0.21 concluímos que $\nu$ se concentra em um único ponto $y \in \bar{\Omega}$, ou seja,

$$
\beta\left(u_{n}\right) \rightarrow \int_{\Omega} x d \nu(x)=y \in \bar{\Omega}
$$

Contradizendo $\operatorname{dist}\left(\beta\left(u_{n}\right), \Omega\right) \nrightarrow 0$.

Lema 2.2.6. $S \doteq m\left(2^{*}, \lambda, \Omega\right)=m\left(2^{*}, \lambda, \mathbb{R}^{N}\right)=S_{2}$.

Demonstração: Primeiramente, mostraremos que $S_{2}=m\left(2^{*}, \lambda, \mathbb{R}^{N}\right)$. Para isso, é suficiente mostrar que $m\left(2^{*}, \lambda, \mathbb{R}^{N}\right) \leqslant S_{2}$. Considere a família

$$
\phi_{\epsilon}(x)=\epsilon^{\frac{2-N}{2}} \phi(x \epsilon), \quad \text { onde } \quad \phi(x)=\frac{[N(N-2)]^{\frac{N-2}{4}}}{\left[1+|x|^{2}\right]^{\frac{N-2}{2}}},
$$

para $\epsilon>0$. É sabido que a melhor constante $S_{2}$ da imersão de Sobolev é atingida pela família $\phi_{\epsilon}$, isto é,

$$
\frac{\int_{\mathbb{R}^{n}}\left|\nabla \phi_{\epsilon}\right|^{2} d x}{\left(\int_{\mathbb{R}^{n}}\left|\phi_{\epsilon}\right|^{2^{*}} d x\right)^{2 / 2^{*}}}=S_{2} .
$$

Portanto, para concluir esse primeiro passo da demonstração, basta verificar que

$$
h(\epsilon) \doteq \frac{\int_{\mathbb{R}^{n}}\left|\phi_{\epsilon}\right|^{2} d x}{\left(\int_{\mathbb{R}^{n}}\left|\phi_{\epsilon}\right|^{*} d x\right)^{2 / 2^{*}}} \rightarrow 0, \quad \text { quando } \epsilon \rightarrow 0 .
$$

De fato, pela definição da função $\phi_{\epsilon}$, após uma mudança de variáveis obtemos

$$
h(\epsilon)=\epsilon^{2} \int_{\mathbb{R}^{n}}|\phi|^{2} d x \rightarrow 0, \quad \text { quando } \epsilon \rightarrow 0 .
$$

Para concluir a demonstração, resta provar que $m\left(2^{*}, \lambda, \mathbb{R}^{N}\right)=m\left(2^{*}, \lambda, \Omega\right)$ para qualquer que seja $\Omega \subset \mathbb{R}^{N}$. É imediato verificar que $m\left(2^{*}, \lambda, \mathbb{R}^{N}\right) \leqslant m\left(2^{*}, \lambda, \Omega\right)$. Reciprocamente, seja $\psi_{m}$ uma sequência minimizante de $m\left(2^{*}, \lambda, \mathbb{R}^{N}\right)$. Pela densidade de $C_{0}^{\infty}\left(\mathbb{R}^{N}\right)$ em $H^{1}\left(\mathbb{R}^{N}\right)$, podemos supor que $\psi_{m} \in C_{0}^{\infty}\left(\mathbb{R}^{N}\right)$. Após uma translação, podemos admitir que $0 \in \Omega$. Agora defina

$$
v_{m}(x)=R_{m}^{-N / 2^{*}} \psi_{m}\left(x / R_{m}\right),
$$

para $R_{m}>0$ suficientemente pequeno de modo que $v_{m} \in C_{0}^{\infty}(\Omega)$. Novamente, para concluir a demonstração, basta mostrar que

$$
g(m) \doteq \frac{\int_{\Omega}\left|v_{m}\right|^{2} d x}{\left(\int_{\Omega}\left|v_{m}\right|^{2^{*}} d x\right)^{2 / 2^{*}}} \rightarrow 0, \quad \text { quando } R_{m} \rightarrow 0 .
$$


De fato, pela definição da função $v_{m}$, após uma mudança de variáveis obtemos

$$
g(m)=R_{m}^{N\left(2^{*}-2\right) / 2} \int_{\Omega / R_{m}}\left|\psi_{m}(y)\right|^{2} d y \rightarrow 0, \quad \text { quando } R_{m} \rightarrow 0 .
$$

Proposição 2.2.7. Para qualquer $\lambda \geqslant 0$ fixado, existe $\bar{p} \equiv \bar{p}(\lambda)$, com $\bar{p} \in\left(2,2^{*}\right)$, tal que para todo $p \in\left[\bar{p}, 2^{*}\right)$,

$$
m(\lambda, p, r)<2^{1-\frac{2}{p}} m(\lambda, p, \Omega)
$$

$e$

$$
\text { se } \Phi_{p, \Omega}(u) \leqslant m(\lambda, p, r) \text {, então } \beta(u) \in \Omega_{r}^{+}, \text {para todo } u \in V_{p}
$$

Demonstração: primeiramente observe que pela escolha de $r$

$$
m(\lambda, p, r)>m(\lambda, p, \Omega)
$$

Assim, o conjunto $\Phi_{p, \Omega}^{c(p, r)}$ é não vazio. Por outro lado, usando a Proposição 2.2.4 e o fato de que $\lim _{p \rightarrow 2^{*}} 2^{1-\frac{2}{p}}=2^{\frac{2}{N}}$ temos

$$
\lim _{p \rightarrow 2^{*}} m(\lambda, p, r)=\lim _{p \rightarrow 2^{*}} m(\lambda, p, \Omega)=S .
$$

Logo, existe $\hat{p} \in\left(2,2^{*}\right)$ tal que (2.21) é satisfeita para todo $p \in\left[\hat{p}, 2^{*}\right)$. Agora observe que pela Proposição 2.2.5 e pelo Lema 2.2.6, existe $\epsilon \in(0,1)$ tal que

$$
\text { se } \frac{\int_{\Omega}\left(|\nabla u|^{2}+\lambda u^{2}\right) d x}{\|u\|_{L^{2^{*}}(\Omega)}^{2}}<S+\epsilon, \text { então } \beta\left(\frac{u}{\|u\|_{L^{2^{*}(\Omega)}}}\right) \in \Omega_{r}^{+} .
$$

Desde modo, fixando $\bar{\epsilon}<\frac{\epsilon}{2(S+1)}$, escolheremos $\bar{p} \in\left[\hat{p}, 2^{*}\right)$ tal que

$$
|m(\lambda, p, r)-S|<\bar{\epsilon},\left.\quad|| \Omega\right|^{\frac{2\left(2^{*}-p\right)}{2^{*} p}}-1 \mid<\bar{\epsilon} \text { para todo } p \in\left[\bar{p}, 2^{*}\right) .
$$

Afirmamos que para todo $p \in\left[\bar{p}, 2^{*}\right)$, 2.22 é satisfeita. De fato, considere $\bar{u} \in H_{0}^{1}(\Omega)$ tal que $\|\bar{u}\|_{L^{p}(\Omega)}=1 \mathrm{e}$

$$
\int_{\Omega}\left(|\nabla \bar{u}|^{2}+\lambda \bar{u}^{2}\right) d x \leqslant m(\lambda, p, r) .
$$

Desde que $\beta\left(\frac{\bar{u}}{\|\bar{u}\|_{L^{2^{*}}(\Omega)}}\right)=\beta(\bar{u})$, obtemos 2.22 de 2.23 se mostrarmos que

$$
\frac{\int_{\Omega}\left(|\nabla \bar{u}|^{2}+\lambda \bar{u}^{2}\right) d x}{\|\bar{u}\|_{L^{2^{*}}(\Omega)}^{2}}<S+\epsilon .
$$


De fato, por (2.11), temos

$$
\begin{aligned}
\frac{\int_{\Omega}\left(|\nabla \bar{u}|^{2}+\lambda \bar{u}^{2}\right) d x}{\|\bar{u}\|_{L^{2^{*}}(\Omega)}^{2}} & \leqslant m(\lambda, p, r)|\Omega|^{\frac{2\left(2^{*}-p\right)}{2^{*} p}} \\
& \leqslant(S+\bar{\epsilon})(1+\bar{\epsilon})<S+\epsilon .
\end{aligned}
$$

Lema 2.2.8. Para todo $r^{\prime}<r$, existe $p\left(r^{\prime}\right) \in\left(1,2^{*}-1\right)$ tal que

$$
c\left(p, r^{\prime}\right)<2 c(p, \Omega) \text { para todo } p \in\left[p\left(r^{\prime}\right), 2^{*}-1\right)
$$

$e$

$$
\beta(u) \in \Omega^{+} \text {para todo } u \in N_{p, \Omega} \operatorname{com} \Phi_{p, \Omega}(u) \leqslant c\left(p, r^{\prime}\right) \text {. }
$$

Demonstração: com os três resultados anteriores mais o Lema B.0.21 do Apêndice B, a demonstração do Lema 2.2 .8 é imediata.

Agora considere a seguinte variedade de Nehari

$$
M_{\lambda, p}=\left\{u \in E \backslash\{0\}: \int_{\mathbb{R}^{N}}\left(|\nabla u|^{2}+(\lambda a(x)+1) u^{2}\right) d x=\int_{\mathbb{R}^{N}}|u|^{p+1} d x\right\}
$$

e defina $c_{\lambda, p}=\inf _{u \in M_{\lambda, p}} \Phi_{\lambda, p}(u)$ onde,

$$
\Phi_{\lambda, p}(u)=\frac{1}{2} \int_{\mathbb{R}^{N}}\left(|\nabla u|^{2}+(\lambda a(x)+1) u^{2}\right) d x-\frac{1}{p+1} \int_{\mathbb{R}^{N}}|u|^{p+1} d x .
$$

Com o objetivo de obter os pontos críticos de $\Phi_{\lambda, p}$ sobre $M_{\lambda, p}$ usaremos o mesmo raciocínio que foi usado em [5] para o problema de Dirichlet e em [22] com o problema de Neumann. Escolheremos $p$ próximo de $2^{*}-1$ de modo que o Lema 1.3.2 seja satisfeito. Para tal $p$, escolheremos $\epsilon>0$ pequeno tal que para $\lambda$ suficientemente grande podemos estimar a categoria de Lusternik-Schnirelmann do conjunto

$$
\Phi_{\lambda, p}^{c_{\lambda, p}+\epsilon}=\left\{u \in M_{\lambda, p}: \Phi_{\lambda, p}(u) \leqslant c_{\lambda, p}+\epsilon\right\}
$$

em termos da categoria de $\Omega$. Finalmente para o $\epsilon>0$ escolhido mostraremos que os pontos críticos neste nível não mudam de sinal. Desde que $H_{0}^{1}(\Omega) \subset E$, então $c_{\lambda, p} \leqslant c(p, \Omega)$.

Corolário 2.2.9. Para todo $p \in\left(1,2^{*}-1\right)$, existe $\Lambda_{0}(p)>0$ tal que $c_{\lambda, p}$ é atingido para todo $\lambda \geqslant \Lambda_{0}(p)$ em algum $u_{\lambda, p}$ que é uma solução não negativa de $\left(S_{\lambda, p}\right)$. 
Lema 2.2.10. $c_{\lambda, p} \rightarrow c(p, \Omega)$ quando $\lambda \rightarrow+\infty$.

Demonstração: observe primeiramente que a desigualdade $c_{\lambda, p}<c(p, \Omega)$ é estrita, pois caso contrário poderíamos encontrar uma solução não negativa de $\left(S_{\lambda, p}\right)$ em $\Omega^{c}$. O que é impossível pelo Princípio do Máximo para as equações elípticas. Admitiremos para a sequência $\lambda_{n} \rightarrow+\infty$ o seguinte limite

$$
\lim _{n \rightarrow+\infty} c_{\lambda_{n}, p}=A<c(p, \Omega)
$$

Observe que $A>0$.

De fato, pelo Corolário 2.2.9 para cada $n$, existe $u_{n} \in M_{\lambda, p}$ tal que

$$
c_{\lambda_{n}, p}=\Phi_{\lambda_{n}, p}\left(u_{n}\right)=\frac{1}{2}\left\|u_{n}\right\|_{\lambda_{n}}-\frac{1}{p+1}\left\|u_{n}\right\|_{L^{p+1}\left(\mathbb{R}^{N}\right)}^{p+1}=\frac{p-1}{2(p+1)}\left\|u_{n}\right\|_{\lambda_{n}}^{2} .
$$

Assim,

$$
\left\|u_{n}\right\|_{\lambda_{n}, p}^{2}=\frac{2(p+1)}{p-1} c_{\lambda_{n}, p} .
$$

Pelo Lema 2.1.2 existe $\sigma>0$ tal que $\sigma^{2}<\|u\|_{\lambda_{n}}^{2}$. Daí,

$$
\sigma^{2} \leqslant \limsup _{n \rightarrow+\infty}\left\|u_{n}\right\|_{\lambda_{n}}^{2}=\frac{2(p+1)}{p-1} A
$$

Portanto, pelo Lema 2.1.3, $A \geqslant c_{0}>0$. Agora pelo Corolário 2.2.9, $c_{\lambda, p}$ é atingido para $\lambda$ grande, assim existe uma sequência $u_{n} \in M_{\lambda, p}$ que são soluções de $\left(S_{\lambda_{n}, p}\right)$ tal que $\Phi_{\lambda_{n}, p}\left(u_{n}\right)=c_{\lambda_{n}, p}$. Desde que $\left(u_{n}\right)$ é limitada em $H^{1}\left(\mathbb{R}^{N}\right)$, podemos admitir $u_{n} \rightarrow u$ em $H^{1}\left(\mathbb{R}^{N}\right)$ e daí,

$$
u_{n} \rightarrow u \text { em } L_{\text {loc }}^{\theta}\left(\mathbb{R}^{N}\right), \text { para } 2 \leqslant \theta<2^{*} .
$$

Afirmamos que $\left.u\right|_{\Omega^{c}}=0$. De fato, suponha $\left.u\right|_{\Omega^{c}} \neq 0$, então existe um conjunto compacto $F \subset \Omega^{c}$ tal que $\operatorname{dist}(F, \Omega)>0,\left.\operatorname{com} u\right|_{F} \neq 0$. Então por (2.24),

$$
\int_{F} u_{n}^{2} d x \rightarrow \int_{F} u^{2} d x>0 .
$$

Mas, desde que $a(x) \geqslant \epsilon_{0}>0$, para todo $x \in F$ segue que

$$
\Phi_{\lambda_{n}, p}\left(u_{n}\right) \geqslant \lambda_{n} \int_{F}\left(a(x) u_{n}^{2}\right) d x \geqslant \lambda_{n} \epsilon_{0} \int_{F} u_{n}^{2} d x \rightarrow+\infty
$$

quando $n \rightarrow+\infty$ o que é um absurdo, pois $\Phi_{\lambda_{n}, p}\left(u_{n}\right) \rightarrow c_{\lambda_{n}, p}$. Logo, $\left.u\right|_{\Omega^{c}}=0$. Agora mostraremos que $u_{n} \rightarrow u$ em $L^{p+1}\left(\mathbb{R}^{N}\right)$. De fato, suponha que não, ou seja, $u_{n} \nrightarrow u$ em $L^{p+1}\left(\mathbb{R}^{N}\right)$. Daí, pelo lema de P. L. Lions [12], existe $\delta_{0}>0, \rho>0 x_{n} \in \mathbb{R}^{N}$, com $\left|x_{n}\right| \rightarrow+\infty$ tal que

$$
\liminf _{n \rightarrow+\infty} \int_{B_{\rho}\left(x_{n}\right)}\left|u_{n}-u\right|^{2} d x \geqslant \delta>0
$$


Portanto,

$$
\begin{aligned}
\Phi_{\lambda_{n}, p}\left(u_{n}\right) & \geqslant \lambda_{n} \int_{B_{\rho}\left(x_{n}\right) \cap\left\{x: a(x)>M_{0}\right\}} a(x) u_{n}^{2} d x=\lambda_{n} \int_{B_{\rho}\left(x_{n}\right) \cap\left\{x: a(x)>M_{0}\right\}} a(x)\left|u_{n}-u\right| d x \\
& \geqslant \lambda_{n} M_{0} \int_{B_{\rho}\left(x_{n}\right)}\left|u_{n}-u\right|^{2} d x-M_{0} \int_{B_{\rho}\left(x_{n}\right) \cap\left\{x: a(x)>M_{0}\right\}}\left|u_{n}-u\right| d x \\
& \geqslant \lambda_{n}\left(M_{0} \int_{B_{\rho}\left(x_{n}\right)}\left|u_{n}-u\right|^{2} d x-o(1)\right) \rightarrow+\infty
\end{aligned}
$$

quando $\lambda_{n} \rightarrow+\infty$. O que é um absurdo. Logo, $u_{n} \rightarrow u$ em $L^{p+1}\left(\mathbb{R}^{N}\right)$. Agora, usando o lema de Fatou segue que

$$
\int_{\mathbb{R}^{N}}\left(|\nabla u|^{2}+u^{2}\right) d x=\int_{\mathbb{R}^{N}}\left(|\nabla u|^{2}+(\lambda a(x)+1) u^{2}\right) d x
$$

pois $\left.u\right|_{\Omega^{c}}=0$ e $a(x)=0 \mathrm{em} \Omega$. Assim,

$$
\begin{aligned}
\int_{\mathbb{R}^{N}}\left(|\nabla u|^{2}(\lambda a(x)+1)+u^{2}\right) d x & \leqslant \limsup _{n \rightarrow+\infty} \int_{\mathbb{R}^{N}}\left(\left|\nabla u_{n}\right|^{2}(\lambda a(x)+1)+u_{n}^{2}\right) d x \\
& =\limsup _{n \rightarrow+\infty} \int_{\mathbb{R}^{N}}\left|u_{n}\right|^{p+1} d x=\int_{\mathbb{R}^{N}}|u|^{p+1} d x
\end{aligned}
$$

Portanto,

Como $\left.u\right|_{\Omega^{c}}=0$, temos

$$
\int_{\mathbb{R}^{N}}\left(|\nabla u|^{2}+u^{2}\right) d x \leqslant \int_{\mathbb{R}^{N}}|u|^{p+1} d x
$$

$$
\int_{\Omega}\left(|\nabla u|^{2}+u^{2}\right) d x \leqslant \int_{\Omega}|u|^{p+1} d x
$$

Assim, existe $\alpha \in M_{\lambda, p}$ tal que

$$
\int_{\Omega}\left(|\nabla(\alpha u)|^{2}+(\alpha u)^{2}\right) d x=\int_{\Omega}|\alpha u|^{p+1} d x
$$

Implicando que

$$
\begin{aligned}
\Phi_{p, \Omega}(\alpha u) & =\frac{p-1}{2(p+1)} \int_{\Omega}\left(|\nabla(\alpha u)|^{2}+(\alpha u)^{2}\right) d x=\frac{p-1}{2(p+1)} \int_{\mathbb{R}^{N}}\left(|\nabla(\alpha u)|^{2}+(\alpha u)^{2}\right) d x \\
& \leqslant \frac{p-1}{2(p+1)} \int_{-}-\mathbb{R}^{N}\left(|\nabla u|^{2}+u^{2}\right) d x \\
& \leqslant \liminf _{n \rightarrow \infty} \frac{p-1}{2(p+1)} \int_{\mathbb{R}^{N}}\left(\left|\nabla u_{n}\right|^{2}+(\lambda a(x)+1) u_{n}^{2}\right) d x=A .
\end{aligned}
$$

Logo, $A \geqslant c(p, \Omega)$. Portanto $\lim _{\lambda \rightarrow \infty} c_{\lambda, p}=c(p, \Omega)$.

Lema 2.2.11. Existe $p_{1} \in\left(1,2^{*}-1\right)$ tal que para todo $p \in\left[p_{1}, 2^{*}-1\right)$, existe $\Lambda_{1}(p) \geqslant \Lambda_{0}(p)$ tal que

$$
c(p, r)<2 c_{\lambda, p} \text { para todo } \lambda \geqslant \Lambda_{1}(p) .
$$


Demonstração: observe que pelo Lema 2.2 .2 temos,

$$
\lim _{p \rightarrow 2^{*}-1} c(p, r)=\lim _{p \rightarrow 2^{*}-1} c(p, \Omega)=\frac{1}{N} S^{\frac{N}{2}} .
$$

Assim, existe $p_{1} \in\left(1,2^{*}-1\right)$ tal que $c(p, r)<2 c(p, \Omega)$ para todo $p \in\left[p_{1}, 2^{*}-1\right)$. Pelo Lema 2.2.10, para cada $p \in\left[p_{1}, 2^{*}-1\right)$, existe $\Lambda_{1}(p)$ tal que

$$
c(p, r)<2 c(p, \Omega) \text {, para todo } \lambda \geqslant \Lambda_{1}(p) .
$$

Agora escolheremos $R_{0}>0$ com $\bar{\Omega} \subset B_{R_{0}}$ e consideremos $\xi: \mathbb{R} \rightarrow \mathbb{R}$ definida por

$$
\xi(t)=1, \text { para } 0 \leqslant t \leqslant R_{0} \quad \text { e } \quad \xi(t)=\frac{R_{0}}{t}, \text { para } R_{0} \leqslant t,
$$

em seguida definimos

$$
\beta_{0}(u)=\frac{\int_{\mathbb{R}^{N}}|u|^{p+1} \xi(|x|) x d x}{\int_{\mathbb{R}^{N}}|u|^{p+1} d x}, \text { para } u \in M_{\lambda, p} .
$$

Lema 2.2.12. Seja $p_{2}=p\left(\frac{r}{2}\right)$ dado pelo Lema 2.2.8. Então para cada $p \in\left[p_{2}, 2^{*}-1\right)$, existe $\Lambda_{2}(p)$ tal que $\beta_{0}(u) \in \Omega^{+}$, para todo $\lambda \geqslant \Lambda_{2}(p)$ e para todo $u \in M_{\lambda, p}$ satisfazendo $\Phi_{\lambda, p}(u) \leqslant c(p, r)$.

Demonstração: suponha por contradição que existam $p \in\left[p_{2}, 2^{*}-1\right), \lambda_{n} \rightarrow+\infty$ e $u_{n} \in M_{\lambda, p}$ tais que $\Phi_{\lambda, p}\left(u_{n}\right) \leqslant c(p, r)$ e $\beta_{0}\left(u_{n}\right) \notin \Omega^{+}$. Como já foi visto anteriormente, a sequência $u_{n}$ é limitada em $H_{0}^{1}\left(\mathbb{R}^{N}\right)$. Deste modo podemos admitir $u_{n} \rightarrow u$ em $H_{0}^{1}\left(\mathbb{R}^{N}\right)$ e daí,

$$
u_{n} \rightarrow u \text { em } L_{\operatorname{loc}}^{\theta}\left(\mathbb{R}^{N}\right) \text { para } 2 \leqslant \theta<2^{*}
$$

Da mesma forma que na demonstração do Lema 2.2.10, podemos mostrar que $\left.u\right|_{\Omega^{c}}=0$. Portanto, usando o fato $\lambda_{n} \rightarrow+\infty$, temos $u_{n} \rightarrow u$ em $L^{2}\left(\mathbb{R}^{N}\right)$. Agora usando a desigualdade de Gagliardo-Nirenberg, temos $u_{n} \rightarrow u$ em $L^{p+1}\left(\mathbb{R}^{N}\right)$.

Desde que $\liminf _{n \rightarrow+\infty} \int_{\mathbb{R}^{N}}\left|\nabla u_{n}\right|^{2} d x \geqslant \int_{\Omega}|\nabla u|^{2} d x$, temos

$$
\int_{\Omega}\left(|\nabla u|^{2}+u^{2}\right) d x \leqslant \int_{\Omega}|u|^{p+1} d x
$$

pois $\left.u\right|_{\Omega^{c}}=0$. Então, existe $\alpha \in(0,1]$ tal que

$$
\int_{\Omega}\left(|\nabla(\alpha u)|^{2}+(\alpha u)^{2}\right) d x=\int_{\Omega}|\alpha u|^{p+1} d x,
$$


ou seja, $\alpha u \in N(p, \Omega)$. Logo,

$$
\begin{aligned}
\Phi_{p, \Omega}(\alpha u) & =\frac{p-1}{2(p+1)} \int_{\Omega}\left(|\nabla(\alpha u)|^{2}+(\alpha u)^{2}\right) d x \leqslant \frac{p-1}{2(p+1)} \int_{\mathbb{R}^{N}}\left(|\nabla u|^{2}+u^{2}\right) d x \\
& \leqslant \liminf _{n \rightarrow+\infty} \frac{p-1}{2(p+1)} \int_{\mathbb{R}^{N}}\left(\left|\nabla u_{n}\right|^{2}+\left(\lambda_{n} a(x)+1\right) u_{n}^{2}\right) d x=\liminf _{n \rightarrow+\infty} \Phi_{\lambda_{n}, p}\left(u_{n}\right) \leqslant c(p, r) .
\end{aligned}
$$

Usando o Lema 2.2 .8 temos $\beta(\alpha u) \in \Omega_{\frac{r}{2}}^{+}$.

Assim,

$$
\beta(u)=\beta(\alpha u) \in \Omega_{\frac{r}{2}}^{+}
$$

Mas, $\lim _{n \rightarrow+\infty} \beta_{0}\left(u_{n}\right)=\beta_{0}(u)=\beta(u)$ e $\beta_{0}\left(u_{n}\right) \notin \Omega_{\frac{r}{2}}^{+}$, o que é uma contradição. Portanto, a conclusão do lema é verdadeira.

Depois de provarmos esses lemas, temos agora os argumentos necessários para demonstrarmos um dos resultados principais deste trabalho.

Demonstração do Teorema 1.0.1: para concluir a prova deste resultado, devemos mostrar que dada uma sequência $\left(u_{n}\right) \subset H^{1}\left(\mathbb{R}^{N}\right), \operatorname{com}\left(u_{n}\right) \subset M_{\lambda_{n}, p}, \Phi_{\lambda_{n}, p}\left(u_{n}\right)=c_{\lambda_{n}, p} \mathrm{e}$ $\lambda_{n} \rightarrow+\infty$, existe uma subsequência convergente em $H^{1}\left(\mathbb{R}^{N}\right)$ para a solução de $(\mathrm{Dp})$ com menor energia. Sabemos que $\left(u_{n}\right)$ é limitada em $H^{1}\left(\mathbb{R}^{N}\right)$, assim $u_{n} \rightarrow u$ em $H^{1}\left(\mathbb{R}^{N}\right)$, e mais, como já foi visto anteriormente, $u_{n} \rightarrow u$ em $L_{\text {loc }}^{\theta}\left(\mathbb{R}^{N}\right)$ para $2 \leqslant \theta<2^{*}$. Vamos mostrar que $u \in H_{0}^{1}(\Omega)$ é a solução com menor energia de $(\mathrm{Dp})$, ou seja $\Phi_{p, \Omega}=c(p, \Omega)$ e $u_{n} \rightarrow u$ em $H^{1}\left(\mathbb{R}^{N}\right)$. Como no Lema 2.2.10, podemos mostrar que $\left.u\right|_{\Omega^{c}}=0$ e $u_{n} \rightarrow u$ em $L^{\theta}\left(\mathbb{R}^{N}\right)$ para $2 \leqslant \theta<2^{*}$. Em consequência, $\Phi_{\lambda, \Omega}(u) v=0$ para todo $v \in H_{0}^{1}(\Omega)$, isto é, $u$ é uma solução fraca de $(\mathrm{Dp})$. Então, para concluir que $u$ é uma solução de energia mínima é suficiente mostrar que

$$
\lambda_{n} \int_{\mathbb{R}^{N}} a(x) u_{n}^{2} d x \rightarrow 0
$$

e

$$
\int_{\mathbb{R}^{N}}\left|\nabla u_{n}\right|^{2} d x \rightarrow \int_{\mathbb{R}^{N}}|\nabla u|^{2} d x .
$$

Para provarmos as convergências acima,vamos usar a prova por absurdo. Suponha,

$$
\limsup _{n \rightarrow+\infty} \lambda_{n} \int_{\mathbb{R}^{N}} a(x) u_{n}^{2} d x>0
$$

$\mathrm{Ou}$

$$
\limsup _{n \rightarrow+\infty} \int_{\mathbb{R}^{N}}\left|\nabla u_{n}\right|^{2} d x>\int_{\mathbb{R}^{N}}|\nabla u|^{2} d x
$$


Seja $\alpha \in(0,1]$ tal que $\alpha u \in M_{\lambda, p}$. Logo,

$$
\begin{aligned}
\Phi_{p, \Omega}(\alpha u) & =\frac{p-1}{2(p+1)} \int_{\Omega}\left(|\nabla(\alpha u)|^{2}+(\alpha u)^{2}\right) d x \\
& <\liminf _{n \rightarrow+\infty} \frac{p-1}{2(p+1)} \int_{\mathbb{R}^{N}}\left(\left|\nabla u_{n}\right|^{2}+\left(\lambda_{n} a(x)+1\right) u_{n}^{2}\right) d x \\
& =\liminf _{n \rightarrow+\infty} \Phi_{\lambda_{n}, p}\left(u_{n}\right)=c(p, \Omega),
\end{aligned}
$$

o que é uma contradição.

Portanto,

$$
\limsup _{n \rightarrow+\infty} \lambda_{n} \int_{\mathbb{R}^{N}}\left(a(x) u_{n}^{2}\right) d x=0
$$

e

$$
\limsup _{n \rightarrow+\infty} \int_{\mathbb{R}^{N}}\left|\nabla u_{n}\right|^{2} d x \rightarrow \int_{\mathbb{R}^{N}}|\nabla u|^{2} d x
$$

Para demonstrarmos o Teorema $\mathbf{1 . 0 . 2}$, precisamos do seguinte resultado.

Lema 2.2.13. Seja u um ponto crítico de $\Phi_{\lambda, p}$ tal que $\Phi_{\lambda, p}(u)<2 c_{\lambda, p}$. Então u nãa muda de sinal.

Demonstração: suponha por contradição que $u$ muda de sinal. Seja $u=u_{+}+u_{-}$, onde $u_{+}=\max \{u, 0\}$ e $u_{-}=\min \{u, 0\}, \operatorname{com} u_{+} \neq 0$ e $u_{-} \neq 0$. Daí,

$$
\int_{\mathbb{R}^{N}}\left(\left|\nabla u_{ \pm}\right|^{2}+(\lambda a(x)+1) u_{ \pm}^{2}\right) d x=\int_{\mathbb{R}^{N}}\left|u_{ \pm}\right|^{p+1} d x
$$

pois $u_{ \pm} \in M_{\lambda, p}$. Portanto,

$$
\Phi_{\lambda}(u)=\Phi_{\lambda}\left(u_{+}\right)+\Phi_{\lambda}\left(u_{-}\right) \geqslant 2 c_{\lambda, p} .
$$

O que é uma contradição, pois por hipótese $\Phi_{\lambda, p}(u)<2 c_{\lambda, p}$.

Para finalizarmos esta seção, demonstraremos os dois últimos teoremas, o Teorema 1.0 .2 e o Teorema 1.0.3.

Demonstração do Teorema 1.0.2; pela escolha de $r$,

$$
c_{\lambda, p}<c(p, \Omega)<c(p, r)
$$

Seja $U \in H_{0}^{1}\left(B_{r}\right)$ a única solução de

$$
\left\{\begin{array}{lr}
-\Delta u+u=u^{p} & \text { em } B_{r}, \\
u=0 & \text { sobre } \partial B_{r} .
\end{array}\right.
$$


Seja também $U_{y}(x)=U(x-y) \in H_{0}^{1}\left(B_{r}(y)\right)$. Então $c(p, r)=\Phi_{p, B_{r}(y)}\left(U_{y}\right)$, para todo $y \in \mathbb{R}^{N}$. Além disso, observe que $U_{y} \in H_{0}^{1}(\Omega)$ e $\Phi_{p, \Omega}\left(U_{y}\right)=c(p, r)$ para todo $y \in \Omega_{r}^{-}$. Considere $p_{0}=\max \left\{p_{1}, p_{2}\right\}$ e $\Lambda(p)=\max \left\{\Lambda_{1}(p), \Lambda_{2}(p)\right\}$ para todo $p \in\left[p_{0}, 2^{*}-1\right)$. Agora defina

$$
\varphi: \Omega_{r}^{-} \rightarrow M_{\lambda, p} \cap \Phi_{\lambda, p}^{c(p, r)}, \varphi(y):=U_{y}
$$

e

$$
\beta_{0}:=\Phi_{\lambda, p}^{c(p, r)} \rightarrow \Omega_{r}^{+} \text {como em } 2.25 .
$$

Observe que o Lema 2.2 .12 nos garante que $\beta_{0}$ e $\varphi$ estão bem definidas. Além disso $\beta \circ \varphi$ é a inclusão $\Omega_{r}^{-} \hookrightarrow \Omega_{r}^{+}$. Vamos definir $Y:=\Phi_{\lambda, p}^{c(p, r)}$ e seja $X:=Y /(-u \sim u)$ espaço quociente de $Y$, onde $u$ e $-u$ são identificados. Claramente $\beta_{0}(-u)=\beta(u)$ o que induz uma aplicação $\beta_{1}: Y \rightarrow \Omega_{r}^{+}$. O funcional $\Phi_{\lambda, p}$ induz um funcional $\Psi: X \rightarrow \mathbb{R}$ com $\psi:=Q \circ \varphi: \Omega_{r}^{-} \rightarrow Y$ onde $Q: X \rightarrow Y$ é a aplicação quociente. Deste modo, pelo Lema C.0.24 do Apêndice $C$ segue que $\operatorname{cat}(X) \geqslant \operatorname{cat}(\Omega)$. Pelo Teorema C.0.26 do Apêndice $\mathrm{C}$, o funcional induzido $\Psi$ possui pelo menos cat $(\Omega)$ pontos críticos, o que corresponde a $\operatorname{cat}(\Omega)$ pares $\pm u_{i}$ de pontos críticos de $\Phi_{\lambda, p}$ abaixo do nível $c(p, r)$. Pelo Lema 2.2.13, as soluções não mudam de sinal e usando o Lema 2.2.11 concluímos que o funcional $\Phi_{\lambda, p}$ tem pelo menos $\operatorname{cat}(\Omega)$ soluções.

Demonstração do Teorema 1.0.3: como já foi visto anteriormente $u_{n}$ é limitada em $H^{1}\left(\mathbb{R}^{N}\right)$, ou seja, a menos de subsequência, $u_{n} \rightarrow u$ em $H^{1}\left(\mathbb{R}^{N}\right)$. Agora do Lema 2.2.10 segue que $u_{n} \in H_{0}^{1}\left(\mathbb{R}^{N}\right)$ e que $u_{n} \rightarrow u$ em $L^{\theta}\left(\mathbb{R}^{N}\right)$ para $2<\theta<2^{*}$.

Desde que $u_{n}$ é solução de $-\Delta u_{n}+(\lambda a(x)+1) u_{n}=u_{n}^{p}$, podemos supor, usando a teoria de regularidade de equações elípticas, que $u_{n} \rightarrow u$ em $C_{l o c}^{2}\left(\mathbb{R}^{N}\right)$. Logo, $u$ é solução de (Dp). Observe que

$$
\begin{aligned}
\int_{\mathbb{R}^{N}}\left|\nabla\left(u_{n}-u\right)\right|^{2} d x & +\int_{\mathbb{R}^{N}}\left(\lambda_{n} a(x)+1\right)\left(u_{n}-u\right)^{2} d x \\
& =\int_{\mathbb{R}^{N}}\left|\nabla u_{n}\right|^{2} d x+\int_{\mathbb{R}^{N}}\left(\lambda_{n} a(x)+1\right) u_{n}^{2} d x-\int_{\mathbb{R}^{N}}|\nabla u|^{2} d x \\
& -\int_{\mathbb{R}^{N}}\left(\lambda_{n} a(x)+1\right) u^{2} d x+o(1) \\
& =\int_{\mathbb{R}^{N}}\left|u_{n}\right|^{p+1} d x-\int_{\mathbb{R}^{N}}|u|^{p+1} d x+o(1)=o(1),
\end{aligned}
$$

ou seja, $u_{n} \rightarrow u$ em $H^{1}\left(\mathbb{R}^{N}\right)$. 


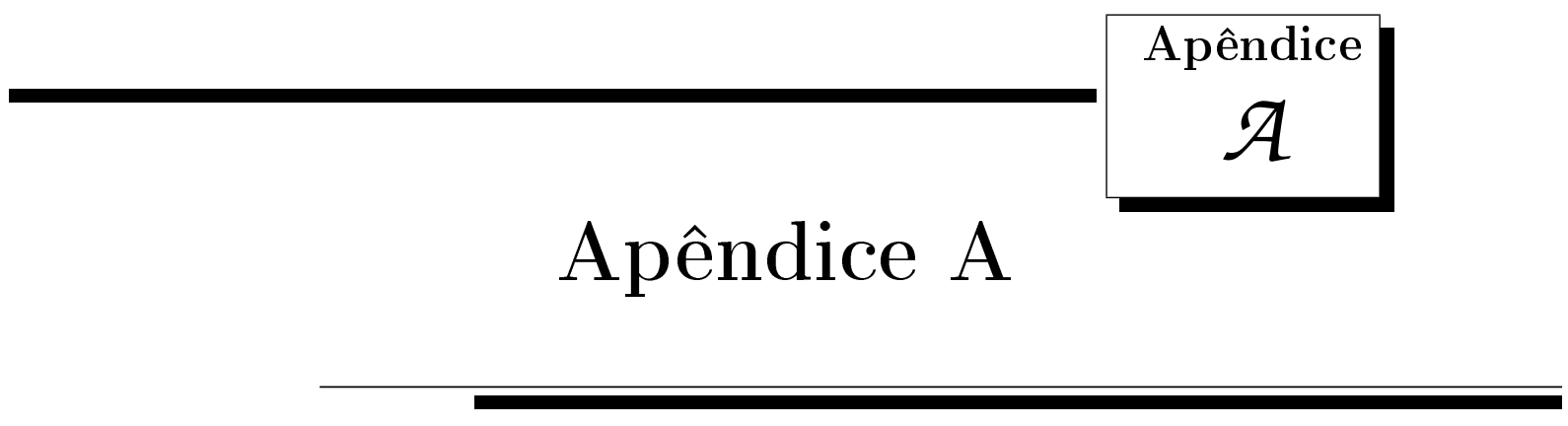

Nesta seção serão enunciados alguns resultados que ajudarão nas demonstrações dos lemas encontrados ao longo deste trabalho. O principal objetivo desta seção é mostrar que funcional $\Phi_{\lambda}$ está bem definido e é de classe $C^{1}$.

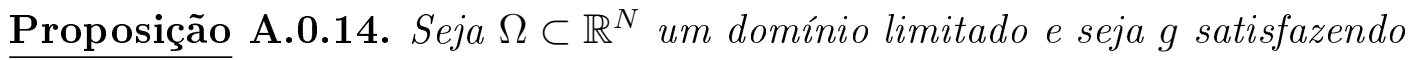

- (i) $g \in C\left(\bar{\Omega} \times \mathbb{R}^{N}, R\right)$;

- (ii) existem constantes $r, s \geqslant 1$ e $a_{1}, a_{2} \geqslant 0$ tais que

$$
|g(x, \xi)| \leqslant a_{1}+a_{2}|\xi|^{\frac{r}{s}}, \text { para todo } x \in \bar{\Omega}, \xi \in \mathbb{R}^{N}
$$

Então a aplicação $\varphi(x) \rightarrow g(x, \varphi(x)) \in C\left(L^{r}(\Omega), L^{s}(\Omega)\right)$

Demonstração: se $u \in L^{r}(\Omega)$, então

$$
\int_{\Omega}|g(x, u(x))|^{s} d x \leqslant \int_{\Omega}\left(a_{1}+a_{2}|u|^{\frac{r}{s}}\right)^{s} d x \leqslant a_{3} \int_{\Omega}\left(1+|u|^{r}\right) d x,
$$

ou seja, $g: L^{r}(\Omega) \rightarrow L^{r}(\Omega)$. Provaremos agora que nossa função $g: L^{r}(\Omega) \rightarrow L^{r}(\Omega)$ é contínua. Observe que $g$ é contínua se, e somente se,

$$
f(x, z(x))=g(x, z(x)+\varphi(x))-g(x, \varphi(x)) \text { é contínua em } z=0 .
$$

Portanto podemos supor $\varphi=0$ e $g(x, 0)=0$. Seja $\epsilon>0$. Afirmamos que existe um $\delta>0$ tal que se $\|u\|_{L^{r}(\Omega)} \leqslant \delta$, então $\|g(\cdot, u)\|_{L^{s}(\Omega)} \leqslant \epsilon$. De fato, por (i) e $g(x, 0)=0$, dado qualquer $\hat{\epsilon}>0$, existe um $\hat{\delta}>0$ tal que $|g(x, \xi)| \leqslant \hat{\epsilon}$ se $x \in \bar{\Omega}$ e $|\xi| \leqslant \delta$. Tome $u \in L^{r}(\Omega)$, $\operatorname{com}\|u\|_{L^{r}(\Omega)} \leqslant \delta$ e seja

$$
\Omega_{1}=\{x \in \bar{\Omega}:|u(x)| \leqslant \beta\} .
$$


Assim,

$$
\int_{\Omega_{1}}|g(x, u(x))|^{s} d x \leqslant \hat{\epsilon}^{s}\left|\Omega_{1}\right| \leqslant \hat{\epsilon}^{s}|\Omega|,
$$

onde $\left|\Omega_{1}\right|$ e $|\Omega|$ denotam a medida de $\Omega_{1}$ e $\Omega$ respectivamente. Escolha $\hat{\epsilon}$ tal que $\hat{\epsilon}^{s} \leqslant\left(\frac{\epsilon}{2}\right)^{s}$. Seja $\Omega_{2}=\bar{\Omega} \backslash \Omega_{1}$. Usando A.1 temos

$$
\int_{\Omega_{2}}|g(x, u(x))|^{s} d x \leqslant \int_{\Omega_{2}}\left(1+|u(x)|^{r}\right) d x \leqslant a_{3}\left(\left|\Omega_{2}\right|+\delta^{r}\right) .
$$

Além disso,

$$
\beta^{r}\left|\Omega_{2}\right| \leqslant \int_{\Omega_{2}}|u(x)|^{r} d x \leqslant \delta^{r}, \quad \text { ou seja } \quad\left|\Omega_{2}\right| \leqslant\left(\delta \beta^{-1}\right)^{r} .
$$

Usando A.3 e A.4 segue que

$$
\int_{\Omega_{2}}|g(x, u(x))|^{s} d x \leqslant a_{3}\left(1+\beta^{-1}\right) \delta^{r} .
$$

Agora escolha $\delta$ tal que $a_{3}\left(1+\beta^{-1}\right) \delta^{r} \leqslant\left(\frac{\epsilon}{2}\right)^{s}$. Logo, usando A.2 e A.5 concluímos que

$$
\|g(\cdot, u)\|_{L^{s}(\Omega)} \leqslant \epsilon \text { se }\|u\|_{L^{r}(\Omega)} \leqslant \delta
$$

Proposição A.0.15. Seja $\Omega \subset \mathbb{R}^{N}$ um dominio limitado com fronteira suave. Seja $p$ uma função satisfazendo

- $\left(p_{1}\right) p(x, \xi) \in C(\bar{\Omega} \times \mathbb{R}, \mathbb{R})$;

- $\left(p_{2}\right)$ existem constantes $a_{1}, a_{2}>0$ tais que

$$
|p(x, \xi)| \leqslant a_{1}+a_{2}|\xi|^{s} \text {, onde } 0 \leqslant s<(N+2)(N-2)^{-1} \text { e } N \geqslant 3 \text {. }
$$

Se

$$
P(x, \xi) \equiv \int_{0}^{\xi} p(x, t) d x
$$

$e$

$$
I(u) \equiv \int_{\Omega}\left(\frac{1}{2}|\nabla u|^{2}-P(x, u)\right) d x,
$$

então $I \in C^{1}\left(H_{0}^{1}(\Omega), \mathbb{R}\right) e$

$$
I^{\prime}(u) \varphi=\int_{\Omega}(\nabla u \nabla \varphi-p(x, u) \varphi) d x, \text { para toda } \varphi \in H_{0}^{1}(\Omega) .
$$

Além disso,

$$
J(u) \equiv \int_{\Omega} P(x, u(x)) d x
$$

é fracamente contínuo e $J^{\prime}(u)$ é compacto. 
Demonstração: usando as imersões de Sobolev, a Proposição A.0.14, $\left(p_{1}\right)$ e $\left(p_{2}\right), I$ está bem definido em $H_{0}^{1}(\Omega)$ e da mesma forma, com a ajuda da desigualdade de Hölder, $I^{\prime}(u)$ também está bem definida. Claramente o primeiro termo de $I$ é de classe $C^{1}$ e o primeiro termo de $I^{\prime}(u)$ é Fréchet diferenciável. Assim, devemos mostrar que

$$
J(u)=\int_{\Omega} P(x, u(x)) d x
$$

com $J(u) \in C^{1}\left(H_{0}^{1}(\Omega), \mathbb{R}\right)$ Faremos isso em duas etapas, primeiro mostraremos que $J$ é Fréchet diferenciável em $H_{0}^{1}(\Omega)$ (por consequência é Lipschitz contínua) e, em seguida , mostraremos que $J^{\prime}(u)$ é contínua. Para começar, seja $u, \varphi \in H_{0}^{1}(\Omega)$. Afirmamos que dado $\epsilon>0$, existe $\delta=\delta(\epsilon, u)$ tal que

$$
|J(u+\varphi)-J(u)-J(u)|=\int_{\Omega} p(x, u) \varphi d x \leqslant \epsilon\|\varphi\|_{H_{0}^{1}(\Omega)} \text {, desde que }\|\varphi\|_{H_{0}^{1}(\Omega)} \leqslant \delta
$$

De fato, considere

$$
\psi \equiv|P(x, u(x)+\varphi(x))-P(x, u(x))-p(x, u(x)) \varphi(x)| .
$$

Daí,

$$
\left|J(u+\varphi)-J(u)-\int_{\Omega} p(x, u) \varphi d x\right| \leqslant \int_{\Omega} \psi d x,
$$

pois

$$
\begin{aligned}
\int_{\Omega} \psi d x & =\int_{\Omega}|P(x, u(x)+\varphi(x))-P(x, u(x))-p(x, u(x)) \varphi(x)| d x \\
& \geqslant\left|\int_{\Omega}(P(x, u(x)+\varphi(x))) d x-\int_{\Omega}(P(x, u(x))) d x-\int_{\Omega}(p(x, u(x)) \varphi(x)) d x\right| \\
& =\left|J(u+\varphi)-J(u)-\int_{\Omega}(p(x, u(x)) \varphi(x)) d x\right| .
\end{aligned}
$$

Agora defina,

$$
\begin{aligned}
& \Omega_{1} \equiv\{x \in \bar{\Omega}:|u(x)| \geqslant \beta\} \\
& \Omega_{2} \equiv\{x \in \bar{\Omega}:|\varphi(x)| \geqslant \gamma\} \\
& \Omega_{3} \equiv\{x \in \bar{\Omega}:|u(x)| \leqslant \beta \text { e }|\varphi(x)| \leqslant \gamma\} .
\end{aligned}
$$

Portanto,

$$
\int_{\Omega} \psi d x \leqslant \sum_{i=1}^{3} \int_{\Omega_{i}} \psi d x .
$$

Pelo Teorema do Valor Médio,

$$
P(x, u+\varphi)-P(x, u)=p(x, u+\theta \varphi) \varphi, \text { onde } \theta \in(0,1) .
$$


Portanto, por A.11), por $\left(p_{2}\right)$ e pela desigualdade de Hölder,

$$
\begin{aligned}
\int_{\Omega_{1}} & |P(x, u(x)+\varphi(x))-P(x, u(x))| d x=\int_{\Omega_{1}}(|p(x, u(x)+\theta \varphi(x))||\varphi(x)|) d x \\
\quad & \leqslant \int_{\Omega_{1}}\left(\left[a_{1}+a_{2}(|u(x)|+|\varphi(x)|)^{s}\right]|\varphi(x)|\right) d x \\
& =\int_{\Omega_{1}} a_{1}|\varphi(x)| d x+\int_{\Omega_{1}} a_{2}(|u(x)|+|\varphi(x)|)^{s}|\varphi(x)| d x \\
& \leqslant a_{1}\left|\Omega_{1}\right|^{\frac{2^{*}-1}{2^{*}}}\|\varphi\|_{L^{2^{*}}\left(\Omega_{1}\right)}+a_{3}\left|\Omega_{1}\right|^{\frac{1}{\sigma}}\left(\|u\|_{L^{s+1}\left(\Omega_{1}\right)}^{s}+\|\varphi\|_{L^{s+1}\left(\Omega_{1}\right)}^{s}\right)\|\varphi\|_{L^{2^{*}}\left(\Omega_{1}\right)}
\end{aligned}
$$

onde $\sigma>1$ é tal que

$$
1=\frac{1}{\sigma}+\frac{s}{s+1}+\frac{N-2}{2 N} .
$$

Após a existência de $\sigma$ é possível $s<2^{*}-1$, ou seja, $\frac{s}{s+1}<\frac{2^{*}-1}{2^{*}}$. Como $\frac{1}{2^{*}}+\frac{2^{*}-1}{2^{*}}=1$, então $\frac{s}{s+1}+\frac{1}{2^{*}}<1$. Assim, existe $\sigma>1$ satisfazendo A.13). Agora, usando (A.12), temos

$$
\int_{\Omega_{1}}|P(x, u(x)+\varphi(x))| d x \leqslant a_{4}\|\varphi\|_{H_{0}^{1}\left(\Omega_{1}\right)}\left(\left|\Omega_{1}\right|^{\frac{2^{*}-1}{2^{*}}}+\left|\Omega_{1}\right|^{\frac{1}{\sigma}}\left(\|u\|_{H_{0}^{1}\left(\Omega_{1}\right)}^{s}+\|\varphi\|_{H_{0}^{1}\left(\Omega_{1}\right)}^{s}\right)\right) .
$$

De forma análoga,

$$
\begin{aligned}
\int_{\Omega_{1}}|p(x, u(x)) \varphi(x)| d x & \leqslant \int_{\Omega_{1}}\left[a_{1}+a_{2}|u(x)|^{s}\right]|\varphi(x)| d x \\
& =\int_{\Omega_{1}} a_{1}|\varphi(x)| d x+\int_{\Omega_{1}} a_{2}|u(x)|^{s}|\varphi(x)| d x \\
& \leqslant a_{1}\left|\Omega_{1}\right|^{\frac{2^{*}-1}{2^{*}}}\|\varphi\|_{L^{2^{*}}\left(\Omega_{1}\right)}+a_{2}\left|\Omega_{1}\right|^{\frac{1}{\sigma}}\|u\|_{L^{s+1}\left(\Omega_{1}\right)}^{s}\|\varphi\|_{L^{2^{*}}\left(\Omega_{1}\right)} \\
& \leqslant a_{4}\|\varphi\|_{H_{0}^{1}(\Omega)}\left(\left|\Omega_{1}\right|^{\frac{2^{*}-1}{2^{*}}}+\left|\Omega_{1}\right|^{\frac{1}{\sigma}}\|u\|_{H_{0}^{1}(\Omega)}^{s}\right) .
\end{aligned}
$$

Observe que

$$
\|u\|_{L^{2}\left(\Omega_{1}\right)}=\left(\int_{\Omega_{1}}|u|^{2} d x\right)^{\frac{1}{2}} \geqslant\left(\int_{\Omega_{1}}|\beta|^{2} d x\right)^{\frac{1}{2}}=\beta\left(\int_{\Omega_{1}} d x\right)^{\frac{1}{2}}=\beta\left|\Omega_{1}\right|^{\frac{1}{2}} .
$$

Assim, usando as imersões de Sobolev e a desigualdade de Hölder, segue que

$$
\|u\|_{H_{0}^{1}(\Omega)} \geqslant a_{6}\|u\|_{L^{2}(\Omega)} \geqslant a_{6}\|u\|_{L^{2}\left(\Omega_{1}\right)} \geqslant a_{6} \beta\left|\Omega_{1}\right|^{\frac{1}{2}}
$$

Portanto,

$$
\left|\Omega_{1}\right|^{\frac{1}{\sigma}} \leqslant\left(\frac{\|u\|_{H_{0}^{1}(\Omega)}}{a_{6} \beta}\right)^{\frac{2}{\sigma}} \equiv M_{1} \text { e }\left|\Omega_{1}\right|^{\frac{2^{*}-1}{2^{*}}} \leqslant\left(\frac{\|u\|_{H_{0}^{1}(\Omega)}}{a_{6} \beta}\right)^{\frac{2\left(2^{*}-1\right)}{2}} \equiv M_{2},
$$


onde $M_{1}, M_{2} \rightarrow 0$, quando $\beta \rightarrow+\infty$.

Agora, por A.12, A.14, A.15 e A.16,

$$
\begin{aligned}
& \int_{\Omega_{1}} \psi d x=\int_{\Omega_{1}}|P(x, u(x)+\varphi(x))-P(x, u(x))-p(x, u(x)) \varphi(x)| d x \\
& \leqslant \int_{\Omega_{1}}|P(x, u(x)+\varphi(x))-P(x, u(x))| d x+\int_{\Omega_{1}}|p(x, u(x)) \varphi(x)| d x \\
& \leqslant a_{4}\|\varphi\|_{H_{0}^{1}(\Omega)}\left[\left|\Omega_{1}\right|^{\frac{2^{*}-1}{2^{*}}}+\left|\Omega_{1}\right|^{\frac{1}{\sigma}}\left(\|u\|_{H_{0}^{1}(\Omega)}^{s}+\|\varphi\|_{H_{0}^{1}(\Omega)}^{s}\right)\right] \\
& +a_{5}\|\varphi\|_{H_{0}^{1}(\Omega)}\left[\left|\Omega_{1}\right|^{\frac{2^{*}-1}{2^{*}}}+\left|\Omega_{1}\right|^{\frac{1}{\sigma}}\|u\|_{H_{0}^{1}(\Omega)}^{s}\right] \\
& \leqslant a_{7}\|\varphi\|_{H_{0}^{1}(\Omega)}\left[\left|\Omega_{1}\right|^{\frac{2^{*}-1}{2^{*}}}+\left|\Omega_{1}\right|^{\frac{1}{\sigma}}\left(\|u\|_{H_{0}^{1}(\Omega)}^{s}+\|\varphi\|_{H_{0}^{1}(\Omega)}^{s}\right)\right] \\
& =a_{7}\|\varphi\|_{H_{0}^{1}(\Omega)}\left[M_{2}+M_{1}\left(\|u\|_{H_{0}^{1}(\Omega)}^{s}+\|\varphi\|_{H_{0}^{1}(\Omega)}^{s}\right)\right] .
\end{aligned}
$$

Logo, podemos supor que $\delta \leqslant 1$. Agora escolha $\beta>0$ tão grande tal que

$$
a_{7}\left[M_{2}+M_{1}\left(\|u\|_{H_{0}^{1}(\Omega)}^{s}+1\right)\right] \leqslant \frac{\epsilon}{3} .
$$

Portanto,

$$
\int_{\Omega_{1}} \psi d x \leqslant \frac{\epsilon}{3}\|\varphi\|_{H_{0}^{1}(\Omega)}
$$

Precisamos fazer uma estimativa para $\int_{\Omega_{2}} \psi d x$, para isso usaremos o mesmo raciocínio. Assim,

$$
\begin{aligned}
\int_{\Omega_{2}} \psi d x & \leqslant a_{3} \int_{\Omega_{2}}\left[1+(|u(x)|+|\varphi(x)|)^{s}\right]|\varphi(x)| d x \\
& \leqslant a_{4}\left\|1+(|u(x)|+|\varphi(x)|)^{s} \mid\right\|_{L^{\frac{s+1}{s}}\left(\Omega_{2}\right)}\|\varphi\|_{L^{s+1}\left(\Omega_{2}\right)} \\
& \leqslant a_{5}\left(1+\|u\|_{H_{0}^{1}(\Omega)}^{s}+\|\varphi\|_{H_{0}^{1}(\Omega)}^{s}\right)\left(\int_{\Omega_{2}}|\varphi(x)|^{s+1}\left(\frac{|\varphi(x)|}{\gamma}\right)^{m-(s+1)} d x\right)^{\frac{1}{s+1}},
\end{aligned}
$$

onde $m=2^{*}-1$. Daí,

$$
\int_{\Omega_{2}} \psi d x \leqslant a_{6} \gamma^{\frac{s+1-m}{s+1}}\left(1+\|u\|_{H_{0}^{1}(\Omega)}^{s}+\|\varphi\|_{H_{0}^{1}(\Omega)}^{s}\right)\|\varphi\|_{H_{0}^{1}(\Omega)}^{\frac{m}{s+1}}
$$

Desde que $P \in C^{1}(\bar{\Omega} \times \mathbb{R}, \mathbb{R})$, dados quaisquer $\hat{\epsilon}, \hat{\beta}>0$, existe $\hat{\gamma}=\hat{\gamma}(\hat{\epsilon}, \hat{\gamma})$ tal que

$$
|P(x, u(x)+\varphi(x))-P(x, u(x))-p(x, u(x)) \varphi(x)| \leqslant \hat{\epsilon}|\varphi(x)|
$$

sempre que $x \in \bar{\Omega},|u| \leqslant \hat{\beta}$ e $|\varphi| \leqslant \hat{\gamma}$. Em particular se $\hat{\beta} \equiv \beta$ e $\gamma \leqslant \hat{\gamma}$, então A.19 e as imersões de Sobolev implicam

$$
\int_{\Omega_{3}} \psi d x \leqslant \hat{\epsilon} \int_{\Omega_{3}}|\varphi(x)| d x \leqslant a_{7} \hat{\epsilon}\|\varphi\|_{H_{0}^{1}(\Omega)} .
$$


Agora, escolha $\hat{\epsilon}$ tal que $3 a_{7} \hat{\epsilon} \leqslant \epsilon$ que determinará $\hat{\gamma}$. Escolha $\gamma=\hat{\gamma}$. Usando A.10, A.18 e A.20 temos

$$
\int_{\Omega} \psi d x \leqslant \frac{2 \epsilon}{3}\|\varphi\|_{H_{0}^{1}(\Omega)}+a_{6} \gamma^{\frac{s+1-m}{s+1}}\left(1+\|u\|_{H_{0}^{1}(\Omega)}^{s}+\|\varphi\|_{H_{0}^{1}(\Omega)}^{s}\right)\|\varphi\|_{H_{0}^{1}(\Omega)}^{\frac{m}{s+1}} .
$$

Finalmente, escolha $\delta$ suficientemente pequeno tal que

$$
a_{6} \gamma^{\frac{1-m}{s+1}}\left(2+\|u\|_{H_{0}^{1}(\Omega)}^{s}\right) \delta^{\frac{m}{s+1}-1} \leqslant \frac{\epsilon}{3}
$$

Dessa forma obtemos A.8. Agora mostraremos o segundo passo, ou seja, que $J^{\prime}(u)$ é contínuo. Para isso, seja $u_{m} \rightarrow u$ em $H_{0}^{1}(\Omega)$. Então, $u_{m} \rightarrow u$ em $L^{s+1}(\Omega)$, pela desigualdade de Hölder e pelas imersões de Sobolev,

$$
\begin{aligned}
\left\|J^{\prime}\left(u_{m}\right)-J^{\prime}(u)\right\|_{H_{0}^{1}(\Omega)} & =\sup _{\|\varphi\| \leqslant 1}\left|\int_{\Omega}\left[P\left(x, u_{m}(x)\right)-p(x, \varphi(x)) \varphi(x)\right] d x\right| \\
& \leqslant a_{8}\left\|P\left(\cdot, u_{m}\right)-P(\cdot, u)\right\|_{L^{s+1}(\Omega)} .
\end{aligned}
$$

Pela hipótese $\left(p_{2}\right),|p(x, u)| \leqslant a_{1}+a_{2}|u|^{\frac{\alpha s}{\alpha}}$, para todo $\alpha \geqslant 1$ e $x \in \bar{\Omega}$, onde $x \in \mathbb{R}$. Daí, pela Proposição A.0.14 temos $p \in C\left(L^{\alpha s}(\Omega), L^{\alpha}(\Omega)\right)$. Escolhendo $\alpha=\frac{s+1}{s}$, podemos ver por A.21 que

$$
\left\|J^{\prime}\left(u_{m}\right)-J^{\prime}(u)\right\| \rightarrow 0, \text { quando } m \rightarrow+\infty
$$

e assim, $J^{\prime}$ é contínuo. Finalmente para mostrar que $J$ é fracamente contínua, seja $u_{m} \rightarrow u$ em $H_{0}^{1}(\Omega)$. Então pelas imersões de Sobolev e pela hipótese $\left(p_{2}\right)$,

$$
u_{m} \rightarrow u \text { em } L^{s+1}(\Omega) \text { desde que } s+1<2^{*} .
$$

Portanto, a Proposição A.0.14 implica que $J\left(u_{m}\right) \rightarrow J(u)$, ou seja $J$ é fracamente contínuo. Como $J$ é fracamente contínuo e uniformemente diferenciável em subconjuntos limitados de $H_{0}^{1}(\Omega)$, concluímos que $J^{\prime}$ é compacto.

Proposição A.0.16. Seja p satisfazendo $\left(p_{1}\right)$ e $\left(p_{2}\right)$ e seja I definido como em (A.6). Se $\left(u_{m}\right)$ é uma sequência limitada em $H_{0}^{1}(\Omega)$ tal que $I^{\prime}\left(u_{m}\right) \rightarrow 0$, quando $m \rightarrow+\infty$, então $\left(u_{m}\right)$ possui uma subsequência convergente.

Demonstração: seja $D: H_{0}^{1}(\Omega) \rightarrow H_{0}^{1}(\Omega)^{*}$ dada por $D(u) \varphi=\int_{\Omega} \nabla u \nabla \varphi d x$. Primeiramente vamos mostrar que $D$ é bijetora.

(i) $D$ é injetora. 
De fato, dados $u, v \in H_{0}^{1}(\Omega)$ tal que $D u=D v$ temos que para todo $\varphi \in H_{0}^{1}(\Omega)$, $(D u) \varphi=(D v) \varphi$, ou seja,

$$
\int_{\Omega} \nabla u \nabla \varphi d x=\int_{\Omega} \nabla v \nabla \varphi d x
$$

o que implica

$$
\int_{\Omega} \nabla(u-v) \varphi d x=0, \text { para todo } \varphi \in H_{0}^{1}(\Omega) .
$$

Logo, $\nabla u=\nabla v$, isto é, $\frac{\partial u}{\partial x_{i}}=\frac{\partial v}{\partial x_{i}}$ (derivada fraca). Então

$$
\int_{\Omega} u \varphi^{\prime} d x=-\int_{\Omega} \frac{\partial u}{\partial x_{i}} \varphi d x=\int_{\Omega} \frac{\partial v}{\partial x_{i}} \varphi d x=\int_{\Omega} v \varphi^{\prime} d x, \quad \text { para todo } \phi \in H_{0}^{1}(\Omega) .
$$

Portanto, $u=v$

(ii) $D$ é sobrejetora.

De fato, seja $f \in H_{0}^{1}(\Omega)^{*}$. Como $H_{0}^{1}(\Omega)$ é um espaço de Hilbert, temos que existe $\varphi \in H_{0}^{1}(\Omega)$ tal que $f(u)=<\varphi, u>$ para todo $u \in H_{0}^{1}(\Omega)$. Assim, pela representação de Riesz,

$$
f(u)=<\varphi, u>=\int_{\Omega} \nabla \varphi \nabla u d x=(D \varphi) u .
$$

Logo, existe $\varphi \in H_{0}^{1}(\Omega)$ tal que $D \varphi=f$.

Assim, de (i) e (ii) segue que $D$ é bijetora, portanto admite inversa $D^{-1}$. Vamos mostrar agora que $D$ é contínua. Seja $u \in E$, temos

$$
\|(D u) \varphi\|=\left|\int_{\Omega} \nabla u \nabla \varphi d x\right| \leqslant \int_{\Omega}\|\nabla u\|\|\nabla \varphi\| d x=\|\nabla u\|_{L^{2}(\Omega)}\|\varphi\|_{L^{2}(\Omega)} \leqslant\|\varphi\|_{H_{0}^{1}(\Omega)} .
$$

Logo, $D u \in E^{*}$, pois $\|\nabla u\|_{L^{2}(\Omega)}$. Assim,

$$
\begin{aligned}
\|D u\|_{H_{0}^{1}\left(\Omega^{*}\right)} & =\sup _{\|\varphi\|=1}\left|\int_{\Omega} \nabla u \nabla \varphi d x\right| \\
& \leqslant \int_{\Omega}\left|\nabla u\|\nabla \varphi \mid d x \leqslant\| \nabla u\left\|_{L^{2}(\Omega)}\right\| \nabla \varphi \|_{L^{2}(\Omega)}\right. \\
& \leqslant\|\nabla u\|_{L^{2}(\Omega)} \leqslant\|u\|_{H_{0}^{1}(\Omega)} .
\end{aligned}
$$

Portanto, $D$ é contínua e, consequentemente, $D^{-1}$ também é contínua.

Como $(D u) \varphi=\int_{\Omega} \nabla u \nabla \varphi d x$, então

$$
D^{-1}\left(I^{\prime}(u)\right)=u-D^{-1}\left[\int_{\Omega} p(x, u) \varphi d x\right]=u-D^{-1}\left(J^{\prime}(u)\right),
$$

pois

$$
J(u)=\int_{\Omega} P(x, u) d x
$$


$\mathrm{e}$

$$
J^{\prime}(u)(v)=\int_{\Omega} p(x, u) v d x
$$

Por A.22,

$$
u_{m}=D^{-1}\left(I^{\prime}\left(u_{m}\right)\right)+D^{-1}\left(J^{\prime}\left(u_{m}\right)\right) .
$$

Por hipótese, $I^{\prime}\left(u_{m}\right) \rightarrow 0$, quando $m \rightarrow+\infty$. Daí, como $u_{m}$ é limitada em $H_{0}^{1}(\Omega)$, e pela proposição A.0.15 $J^{\prime}$ é compacto, então $J^{\prime}\left(u_{m}\right)$ admite uma subsequência convergente. Logo,

$$
u_{m_{j}}=D^{-1}\left(I^{\prime}\left(u_{m_{j}}\right)\right)+D^{-1}\left(J^{\prime}\left(u_{m_{j}}\right)\right) \rightarrow u,
$$

onde $u_{m_{j}}$ é uma subsequência de $u_{m}$. Portanto, $u_{m}$ admite uma subsequência convergente.

Observe que os resultados acima são válidos para $\Omega$ limitado, mas em nosso principal resultado trabalhamos com $\Omega=\mathbb{R}^{N}$. Portanto, precisamos também dos dois próximos resultados.

Definição A.0.17. Seja $\Omega \subset \mathbb{R}^{N}$ um dominio com medida de Lebesgue infinita. Sobre o espaço $L^{p}(\Omega) \cap L^{q}(\Omega)$ definimos a seguinte norma

$$
|u|_{p \wedge q}:=\|u\|_{L^{p}(\Omega)}+\|u\|_{L^{q}(\Omega)}
$$

e sobre o espaço $L^{p}(\Omega)+L^{q}(\Omega)$ definimos a norma

$$
|u|_{p \vee q}:=\inf \left\{\|v\|_{L^{p}(\Omega)}+\|w\|_{L^{q}(\Omega)}: v \in L^{p}(\Omega), w \in L^{q}(\Omega), u=v+w\right\} .
$$

Teorema A.0.18. Sejam $1 \leqslant p, q, r, s<\infty, f \in C(\bar{\Omega} \times \mathbb{R}) e$

$$
|f(x, u)| \leqslant c\left(|u|^{\frac{p}{r}}+|u|^{\frac{q}{s}}\right) .
$$

Então para todo $u \in L^{p}(\Omega) \cap L^{q}(\Omega)$, temos $f(\cdot, u) \in L^{r}(\Omega)+L^{s}(\Omega)$ e o operador

$$
T: L^{p}(\Omega) \cap L^{q}(\Omega) \rightarrow L^{r}(\Omega)+L^{s}(\Omega): u \mapsto f(x, u)
$$

é contínuo.

Demonstração: seja $\psi \in D(]-2,2[)$ tal que $\psi=1$ em $]-1,1[$ e defina

$$
g(x, u):=\psi(u) f(x, u) \text { e } h(x, u):=(1-\psi(u)) f(x, u)
$$

Sem perda de generalidade, considere $\frac{p}{r} \leqslant \frac{q}{s}$. Deste modo obtemos

$$
|g(x, u)| \leqslant a|u|^{\frac{p}{r}} \text { e }|h(x, u)| \leqslant b|u|^{\frac{q}{s}} .
$$


Usando A.23), podemos supor que $u_{n} \rightarrow u$ em $L^{p}(\Omega) \cap L^{q}(\Omega)$. Assim, $g\left(x, u_{n}\right) \rightarrow g(x, u)$ em $L^{r}(\Omega)$ e $h\left(x, u_{n}\right) \rightarrow h(x, u)$ em $L^{s}(\Omega)$. Pela definição da norma $|\cdot|_{p \wedge q}$,

$$
\left|f\left(x, u_{n}\right)-f(x, u)\right|_{r \vee s} \leqslant\left\|g\left(x, u_{n}\right)-g(x, u)\right\|_{L^{r}(\Omega)}+\left\|h\left(x, u_{n}\right)-h(x, u)\right\|_{L^{s}(\Omega)} .
$$

Portanto, $f\left(x, u_{n}\right) \rightarrow f(x, u)$ em $L^{r}(\Omega)+L^{s}(\Omega)$, ou seja, o operador $T$ é contínuo.


$C^{1}(E, \mathbb{R})$.

Demonstração: para mostrarmos que $\Phi_{\lambda} \in C^{1}(E, \mathbb{R})$, mostraremos que a derivada de Gateaux de $\psi(u)=\int_{\mathbb{R}^{N}} F(x, u) d x$, onde $F(x, u):=\int_{0}^{u} f(x, s) d s$, existe e é contínua.

(i) Existência da derivada de Gateaux.

Sejam $u, h \in H^{1}\left(\mathbb{R}^{N}\right)$. Dados, $x \in \mathbb{R}^{N}$ e $0<|t|<1$, pelo Teorema do Valor Médio, existe $\lambda \in] 0,1[$ tal que

$$
\begin{aligned}
\frac{|F(x, u(x)+t h(x))-F(x, u(x))|}{|t|} & =|(f(x, u(x)+\lambda t h(x))) h(x)| \\
& \leqslant c(|u(x)|+|h(x)|)+2^{p-1}\left(|u(x)|^{p-1}+|h(x)|^{p-1}\right)|h(x)| .
\end{aligned}
$$

A desigualdade de Hölder implica que,

$$
\left((|u(x)|+|h(x)|)+2^{p-1}\left(|u(x)|^{p-1}+|h(x)|^{p-1}\right)|h(x)|\right) \in L^{1}\left(\mathbb{R}^{N}\right) .
$$

Pelo Teorema da Convergência Dominada de Lebesgue temos

$$
<\psi^{\prime}(u), h>=\int f(x, u) h d x .
$$

(ii) Continuidade da derivada de Gateaux.

Considere $u_{n}$ tal que $u_{n} \rightarrow u$ em $H^{1}\left(\mathbb{R}^{N}\right)$. Pelas imersões de Sobolev, podemos admitir que $u_{n} \rightarrow u$ em $L^{2}\left(\mathbb{R}^{N}\right) \cap L^{q}\left(\mathbb{R}^{N}\right)$. Segue do Terorema A.0.18 que $f\left(x, u_{n}\right) \rightarrow f(x, u)$ em $L^{2}\left(\mathbb{R}^{N}\right)+L^{q}\left(\mathbb{R}^{N}\right)$, onde $q:=\frac{p}{p-1}$. Logo, usando novamente a desigualdade de Hölder, obteremos

$$
\begin{aligned}
\left|<\psi^{\prime}\left(u_{n}\right)-\psi^{\prime}(u), h>\right| & \leqslant\left|f\left(x, u_{n}\right)-f(x, u)\right|_{2 \vee q}|h|_{2 \wedge p} \\
& \leqslant c_{p}\left|f\left(x, u_{n}\right)-f(x, u)\right|_{2 \vee q}\|h\|_{L^{1}\left(\mathbb{R}^{N}\right)}
\end{aligned}
$$

e então,

$$
\left\|\psi^{\prime}\left(u_{n}\right)-\psi^{\prime}(u)\right\| \leqslant c_{p}\left|f\left(x, u_{n}\right)-f(x, u)\right|_{2 \vee q} \rightarrow 0 \text { quando } n \rightarrow+\infty
$$

Assim, de (i) e (ii) segue que $\Phi_{\lambda} \in C^{1}\left(E, \mathbb{R}^{N}\right)$. 


\section{Apêndice}

\section{Apêndice B}

Ao longo deste trabalho usamos muitos resultados clássicos de espaços $L^{p}$ e espaços de Hilbert, a maioria dos resultados pode ser encontrados em [Bre83]. Nesta seção vamos enunciar alguns resultados utilizados nas demonstrações dos lemas e proposições encontrados neste trabalho.

$$
\begin{aligned}
\text { Seja } N \geqslant 3 \text { e } 2^{*}= & \frac{2 N}{N-2} \text {. O espaço } \\
& D^{1,2}\left(\mathbb{R}^{N}\right)=\left\{u \in L^{2^{*}}\left(\mathbb{R}^{N}\right):|\nabla u| \in L^{2}\left(\mathbb{R}^{N}\right)\right\}
\end{aligned}
$$

com o produto interno

$$
\int_{\mathbb{R}^{N}} \nabla u \nabla v d x
$$

e a correspondente norma

$$
\left(\int_{\mathbb{R}^{N}}|\nabla u|^{2} d x\right)^{\frac{1}{2}}
$$

é um espaço de Hilbert. O espaço $D_{0}^{1,2}(\Omega)$ é definido como o fecho de $D(\Omega)$ (o espaço das funções $C^{\infty}$ com suporte compacto em $\Omega$ ) em $D^{1,2}\left(\mathbb{R}^{N}\right)$. O próximo teorema estabelece algumas das imersões de Sobolev utilizadas neste trabalho, cujas demonstrações podem ser encontradas em [Bre83].

Teorema B.0.20. As seguintes imersões são contínuas:

$$
\begin{array}{ll}
H^{1}\left(\mathbb{R}^{N}\right) \subset L^{p}\left(\mathbb{R}^{N}\right), & 2 \leqslant p<+\infty, N=1,2 ; \\
H^{1}\left(\mathbb{R}^{N}\right) \subset L^{q}\left(\mathbb{R}^{N}\right), & 2 \leqslant q \leqslant 2^{*}, N \leqslant 3 \\
D^{1,2}\left(\mathbb{R}^{N}\right) \subset L^{2^{*}}\left(\mathbb{R}^{N}\right), & N \geqslant 3 .
\end{array}
$$

Denotamos por $S_{2}$ a melhor constante de Sobolev da imersão $D^{1,2}\left(\mathbb{R}^{N}\right) \subset L^{2^{*}}\left(\mathbb{R}^{N}\right)$, isto é,

$$
S_{2}=\inf \left\{\int_{\mathbb{R}^{N}}|\nabla u|^{2} d x:\|u\|_{L^{2^{*}\left(\mathbb{R}^{N}\right)}}=1\right\}
$$


Além disso, é válido que $S_{2}>0$. Para contornar a perda de compacidade das imersões de Sobolev, citadas no teorema anterior, utilizamos o seguinte teorema de P. L. Lions na prova dos resultados principais. A demonstração do teorema é técnica e por isso será omitida. Uma prova pode ser encontrada em [Wil96] ou em [Lio84].

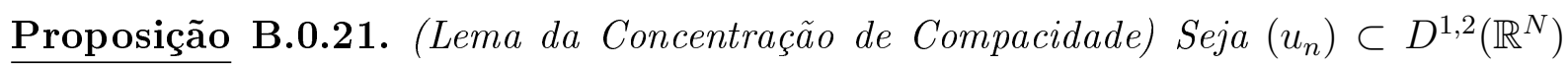
uma sequência tal que

$$
\begin{array}{lc}
u_{n} \rightarrow u & \text { em } D^{1,2}\left(\mathbb{R}^{N}\right), \\
\left|\nabla\left(u_{n}-u\right)\right|^{2} \rightarrow \mu & \text { em } M\left(\mathbb{R}^{N}\right), \\
\left|u_{n}-u\right|^{2^{*}} \rightarrow \nu & \text { em } M\left(\mathbb{R}^{N}\right), \\
u_{n} \rightarrow u & \text { qtp em } \mathbb{R}^{N} .
\end{array}
$$

e defina

$$
\mu_{\infty}:=\lim _{R \rightarrow+\infty} \limsup _{n \rightarrow+\infty} \int_{|x| \leqslant R}\left|\nabla u_{n}\right|^{2} d x, \quad \nu_{\infty}:=\lim _{R \rightarrow+\infty} \limsup _{n \rightarrow+\infty} \int_{|x|>R}\left|u_{n}\right|^{2^{*}} d x .
$$

Então

$$
\begin{gathered}
\|\nu\|^{\frac{2}{2^{*}}} \leqslant S_{2}^{-1}\|\mu\|, \\
\nu_{\infty}^{\frac{2}{2^{*}}} \leqslant S_{2}^{-1} \mu_{\infty}, \\
\limsup _{n \rightarrow+\infty}\left\|\nabla u_{n}\right\|_{L^{2}\left(\mathbb{R}^{N}\right)}^{2}=\|\nabla u\|_{L^{2}\left(\mathbb{R}^{N}\right)}^{2}+\|\mu\|+\mu_{\infty}, \\
\limsup _{n \rightarrow+\infty}\left\|u_{n}\right\|_{L^{2^{*}\left(\mathbb{R}^{N}\right)}}^{2^{*}}=\|u\|_{L^{2^{*}\left(\mathbb{R}^{N}\right)}}^{2^{*}}+\|\nu\|+\nu_{\infty} .
\end{gathered}
$$

Além disso, se $u=0$ e $\|\nu\|^{\frac{2}{2^{*}}}=S_{2}^{-1}\|\mu\|$, então $\nu$ e $\mu$ estão concentradas em um único ponto, onde $M\left(\mathbb{R}^{N}\right)$ denota o espaço das medidas finitas em $\mathbb{R}^{N}$.

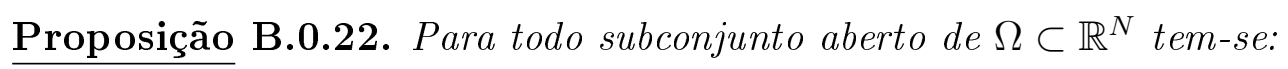

- $S_{2}(\Omega):=\inf _{u \in D_{0}^{1,2}(\Omega)}\left\{\|\nabla u\|_{L^{2}(\Omega)}^{2}:\|u\|_{L^{2^{*}}(\Omega)}=1\right\}=S_{2}$,

- $S_{2}(\Omega)$ nunca é atingido, exceto quando $\Omega=\mathbb{R}^{N}$. 


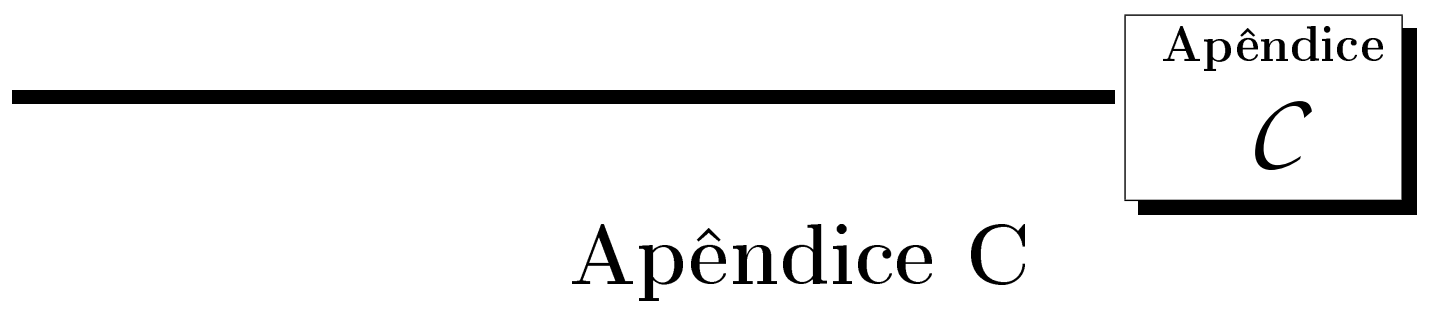

Agora enunciaremos alguns resultados envolvendo categoria de um conjunto, cujas demonstrações podem ser encontradas em Wil96.

Definição C.0.23 (Categoria de Lusternik e Schnirelman). Sejam X um espaço topológico e $A \subset X$ um fechado. A categoria de Lusternik e Schnirelman (LS) de A com respeito a $X$, denotada por $\operatorname{cat}_{X}(A)$, é o menor inteiro $n$ tal que

$$
A \subset A_{1} \ldots A_{n}
$$

com $A_{i}$ fechado e contrátil em $X$, para cada $i=1, \ldots, n$. Lembrando que um subconjunto $A$ de um espaço topológico $X$ é contrátil em $X$ se a inclusão $i: A \hookrightarrow X$ é homotópica a uma constante $p \in X$, ou seja, se existe $H \in C([0,1] \times A, X)$ tal que $H(0, u)=u$ e $H(1, u)=p$.

Lema C.0.24. Sejam $A, B$ e $X$ espaços topológicos e suponha que exista $\psi: A \hookrightarrow X e$ $\beta: X \rightarrow B$ tal que $\beta \circ \psi: A \rightarrow B$ é uma homotopia equivalente. Então cat $(X) \geqslant \operatorname{cat}(A)$.

A demonstração deste lema pode ser encontrada em [BW95.

Teorema C.0.25. Se $\left.\varphi\right|_{V}$ satisfaz $(P S)_{c}$, onde

$$
a=\sup _{Y} \varphi<c \doteq c_{k}=c_{k+1}=\cdots=c_{k+m} \leq d,
$$

então

$$
\operatorname{cat}_{\varphi^{d}}\left(K_{c}\right) \geq m+1
$$

Teorema C.0.26. Se $\left.\varphi\right|_{V}$ é limitado inferiormente e satisfaz $(P S)_{c}$ para todo $c \in$ $\left[\inf _{V} \varphi, d\right]$, então $\left.\varphi\right|_{V}$ possui pelo menos $\operatorname{cat}_{\varphi^{d}}\left(\varphi^{d}\right)$ pontos críticos e $\left.\varphi\right|_{V}$ tem um mínimo. 


\section{Referências Bibliográficas}

[AdMFS09] Claudianor O. Alves, Daniel C. de Morais Filho, and Marco A. S. Souto, Multiplicity of positive solutions for a class of problems with critical growth in $\mathbb{R}^{N}$, Proc. Edinb. Math. Soc. (2) 52 (2009), no. 1, 1-21. MR MR2475877

[AS08] Claudianor O. Alves and Marco A. S. Souto, Multiplicity of positive solutions for a class of problems with exponential critical growth in $\mathbb{R}^{2}$, J. Differential Equations 244 (2008), no. 6, 1502-1520. MR MR2396507 (2008m:35088)

[BC91] Vieri Benci and Giovanna Cerami, The effect of the domain topology on the number of positive solutions of nonlinear elliptic problems, Arch. Rational Mech. Anal. 114 (1991), no. 1, 79-93. MR MR1088278 (91j:35102)

[Bre83] Haïm Brezis, Analyse fonctionnelle, Collection Mathématiques Appliquées pour la Maîtrise. [Collection of Applied Mathematics for the Master's Degree], Masson, Paris, 1983, Théorie et applications. [Theory and applications]. MR MR697382 (85a:46001)

[BW95] Thomas Bartsch and Zhi Qiang Wang, Existence and multiplicity results for some superlinear elliptic problems on $\mathbf{R}^{N}$, Comm. Partial Differential Equations 20 (1995), no. 9-10, 1725-1741. MR MR1349229 (96f:35050)

[BW00] Thomas Bartsch and Zhi-Qiang Wang, Multiple positive solutions for a nonlinear Schrödinger equation, Z. Angew. Math. Phys. 51 (2000), no. 3, 366-384. MR MR1762697 (2001f:35363)

[CW07] Jan Chabrowski and Zhi-Qiang Wang, Exterior nonlinear Neumann problem, NoDEA Nonlinear Differential Equations Appl. 13 (2007), no. 5-6, 683-697. MR MR2329024 (2008g:35051) 
[dD02] D. G. deFigueiredo and Y. H. Ding, Solutions of a nonlinear Schrödinger equation, Discrete Contin. Dyn. Syst. 8 (2002), no. 3, 563-584. MR MR1897867 (2003c:35038)

[DS07] Yanheng Ding and Andrzej Szulkin, Bound states for semilinear Schrödinger equations with sign-changing potential, Calc. Var. Partial Differential Equations 29 (2007), no. 3, 397-419. MR MR2321894 (2008c:35067)

[Lio84] P.-L. Lions, The concentration-compactness principle in the calculus of variations. The locally compact case. I, Ann. Inst. H. Poincaré Anal. Non Linéaire 1 (1984), no. 2, 109-145. MR MR778970 (87e:49035a)

[Wi196] Michel Willem, Minimax theorems, Progress in Nonlinear Differential Equations and their Applications, 24, Birkhäuser Boston Inc., Boston, MA, 1996. MR MR1400007 (97h:58037) 